ARTICLE

Received 20 Sep 2015 | Accepted 30 Sep 2016 | Published 11 Nov 2016

DOI: $10.1038 /$ ncomms13412

OPEN

\title{
Arabidopsis seed germination speed is controlled by SNL histone deacetylase-binding factor-mediated regulation of $A \cup X 1$
}

\author{
Zhi Wang ${ }^{1}$, Fengying Chen ${ }^{1}$, Xiaoying $\mathrm{Li}^{1,2}$, Hong Cao ${ }^{1}$, Meng Ding ${ }^{1,2}$, Cun Zhang ${ }^{1,2}$, Jinghong Zuo ${ }^{1,2}$, \\ Chaonan $\mathrm{Xu}^{1,2}$, Jimei $\mathrm{Xu}^{1,2}$, Xin Deng ${ }^{3}$, Yong Xiang ${ }^{4}$, Wim J.J. Soppe ${ }^{4,5} \&$ Yongxiu Liu ${ }^{1}$
}

Histone acetylation is known to affect the speed of seed germination, but the molecular regulatory basis of this remains ambiguous. Here we report that loss of function of two histone deacetylase-binding factors, SWI-INDEPENDENT3 (SIN3)-LIKE1 (SNL1) and SNL2, results in accelerated radicle protrusion and growth during seed germination. AUXIN RESISTANT 1 (AUX1) is identified as a key factor in this process, enhancing germination speed downstream of SNL1 and SNL2. AUX1 expression and histone H3 acetylation at lysines 9 and 18 is regulated by SNL1 and SNL2. The D-type cyclins encoding genes CYCD1;1 and CYCD4;1 display increased expression in AUX1 over-expression lines and the snl1sn/2 double mutant. Accordingly, knockout of CYCD4;1 reduces seed germination speed of AUX1 over-expression lines and snl7snl2 suggesting the importance of cell cycling for radicle protrusion during seed germination. Together, our work identifies AUX1 as a link between histone acetylation mediated by SNL1 and SNL2, and radicle growth promoted by CYCD1;1 and CYCD4;1 during seed germination.

\footnotetext{
${ }^{1}$ Key Laboratory of Plant Molecular Physiology, Institute of Botany, Chinese Academy of Sciences, Beijing 100093, China. ${ }^{2}$ College of Life Sciences, University of Chinese Academy of Sciences, Beijing 100049, China. ${ }^{3}$ Key Laboratory of Plant Resources, Institute of Botany, Chinese Academy of Sciences, Beijing 100093 , China. ${ }^{4}$ Department of Plant Breeding and Genetics, Max Planck Institute for Plant Breeding Research, 50829 Cologne, Germany. ${ }^{5}$ Institute of Molecular Physiology and Biotechnology of Plants (IMBIO), University of Bonn, 53115 Bonn, Germany. Correspondence and requests for materials should be addressed to Y.L. (email: yongxiu@ibcas.ac.cn).
} 
G ermination is a critical step in the life cycle of seed plants converting a quiescent seed to a highly active seedling. Seed germination is required for the next generation to enter the ecosystem, and its proper timing ensures offspring propagate under suitable conditions. In agriculture, fast and uniform seed germination is also necessary for high crop yield. Seeds can germinate after the release of dormancy by extended storage (after-ripening) or imbibition at species-specific temperatures (stratification). Germination includes a subsequent series of events starting with the uptake of water by the dry seed and finishing with the elongation of the embryonic axis and the protrusion of the radicle ${ }^{1}$. Seed germination is a complex process regulated by genetic and environmental factor ${ }^{2-5}$.

Studies have identified crucial roles for abscisic acid (ABA) and gibberellic acid (GA) in seed germination ${ }^{6,7}$. The application of exogenous $\mathrm{ABA}$ inhibits seed germination and mutants defective in $\mathrm{ABA}$ biosynthesis or signalling have enhanced germination efficiency ${ }^{6,7}$. The ABSCISIC ACID INSENSITIVE (ABI) factors, $\mathrm{ABI}, \mathrm{ABI}, \mathrm{ABI}, \mathrm{ABI} 4$ and $\mathrm{ABI}$, act in the $\mathrm{ABA}$ inhibition of seed germination ${ }^{8,9}$. Conversely, GA promotes seed germination. GA-deficient mutants such as ga1 and ga2 show a delay or absence of seed germination ${ }^{10,11}$. GA signalling requires the DELLA proteins REPRESSOR OF GA (RGA), GIBBERELLIC ACID INSENSITIVE (GAI) and RGA-LIKE 2 (RGL2), which play negative roles in seed germination ${ }^{6,12,13}$.

Apart from ABA and GA additional hormones like auxin play a role in germination ${ }^{5}$. Auxin has been shown to function both positively and negatively in seed germination depending on its dose. Exogenous application of high auxin concentrations from 0.3 to $1 \mu \mathrm{m}$ indole-3-acetic acid (IAA) can inhibit seed germination in Arabidopsis ${ }^{14,15}$. In contrast application of low concentrations of auxin from 0.03 to $3 \mathrm{nM}$ IAA can promote seed germination and seedling establishment ${ }^{16,17}$. Genetic studies have also provided evidence for the involvement of auxin pathway factors in both germination completion and early seedling establishment ${ }^{18,19}$. AUXIN RESPONSE FACTORS (ARF) $10 / 16$ act as positive regulators of the ABA signal pathway by regulating expression of $A B I 3$, and the arf10arf 16 double mutant showed insensitivity for seed germination to $\mathrm{ABA}^{18}$. Transgenic seeds expressing a miR160-resistant form of ARF10 (mARF10) were hypersensitive to germination inhibition by exogenous $\mathrm{ABA}$, whereas ectopic expression of miR160 resulted in a reduced sensitivity to $\mathrm{ABA}^{15}$. Interestingly, another $A R F$ gene, $A R F 2$, was induced by $A B A$, and the arf2 mutants displayed enhanced $\mathrm{ABA}$ sensitivity during seed germination. Conversely, over-expression of $A R F 2$ decreased the inhibition of seed germination by $A B A^{19}$, suggesting that ARF2 is involved in seed germination by repressing the ABA signalling pathway. Transcriptomic studies have shown that RNAs encoding the auxin transporters AUXIN RESISTANT 1 (AUX1), PIN-FORMED 2 (PIN2) and PIN7 were highly upregulated in response to GA treatment of gal mutant seeds $^{20}$. In addition, both efflux and influx transporters are upregulated in after-ripened seeds compared with dormant seeds $^{21}$, suggesting that auxin transporters might be important for seed germination. AUX1 is required for ABA inhibition of seed germination, loss-of-function mutants of AUX1 showed increased $\mathrm{ABA}$ resistance ${ }^{22}$. These results indicate that distinct auxin signalling pathways are involved in seed germination by affecting $\mathrm{ABA}$ and/or GA signal pathways. These functions of auxin are commonly achieved through the auxin transport carriers in the root tip among which AUX1 has an important role ${ }^{23}$.

Apart from plant hormones, chromatin factors have been shown to control seed germination. PICKLE (PKL), a CHD3 class SWI/SNF chromatin-remodelling factor, is involved in repression of embryonic traits during germination. $P K L$ transcript is absent in dry seeds and is initiated on seed imbibition ${ }^{24}$. The $p k l$ mutants showed hypersensitivity to ABA-mediated repression of germination, indicating that PKL acts as a negative factor of ABA signalling during seed germination ${ }^{25}$. Mutants in FERTILIZATION-INDEPENDENT ENDOSPERM, an essential component of the polycomb repressive complex 2 , display a genome-wide reduction in histone 3 lysine 27 trimethylation (H3K27me3) and exhibit increased seed germination defects $^{26}$. Mutations in KRYPTONITE/SU(VAR)3-9 HOMOLOG4 (KYP/SUVH4), encoding a histone methyltransferase that is required for histone 3 lysine 9 dimethylation (H3K9me2) ${ }^{27}$, result in decreased seed germination in response to $\mathrm{ABA}$ and the GA biosynthesis inhibitor paclobutrazol ${ }^{28}$. The histone arginine demethylases, JUMONJI DOMAIN-CONTAINING PROTEIN 20 (JMJ20) and JMJ22, act redundantly as positive regulators of seed germination by removing repressive histone arginine methylation at GA3ox1/GA3ox2 to regulate GA levels ${ }^{29}$. Arabidopsis AL PHD-PRC1 complexes have been shown to promote seed germination by switching the chromatin state from H3K4me3 to H3K27me3 to repress seed developmental genes such as ABI3, DELAY OF GERMINATION 1 (DOG1) and $\mathrm{CHOTTO1}(\mathrm{CHO})^{30}$. Recently, a role for histone acetylation in seed germination was demonstrated by the increased seed germination speed phenotype of the histone deacetylase 9 ( $h d a 9$ ) mutant $^{31}$.

We previously showed that histone deacetylation mediated by SNL1 and SNL2 is involved in the regulation of seed dormancy by affecting the $\mathrm{ABA} /$ ethylene antagonism ${ }^{32}$. Here we report that SNL1 and SNL2 also regulate radicle promotion and early growth in a manner dependent on AUX1. We show that SNLs repress AUX1 expression and histone deacetylation of H3K9K18ac. Loss of function of SNL1 and SNL2 causes significantly increased transcript levels of auxin-related genes, including AUX1. Genetic evidence further confirms that AUX1 is a key factor with a positive role in regulating seed germination downstream of SNL1/SNL2. Moreover, we identify potentially important roles for the D-type cyclins CYCD1;1 and CYCD4;1 in radicle protrusion and early growth mediated by AUX1 and SNL1/SNL2.

\section{Results}

SNL1 and SNL2 negatively regulate radicle protrusion. We have previously shown that SNL1 and SNL2 are components of the SNL-HDA19 histone deacetylation complex and that they regulate seed dormancy by deacetylating histone $\mathrm{H} 3 \mathrm{~K} 9 / 18$ sites of $\mathrm{ABA}$ and ethylene signalling-related genes ${ }^{32}$. We analysed seed germination of snl1, snl2 single and snllsnl2 double mutants in more detail and observed accelerated radicle protrusion and growth of afterripened seeds with fully released seed dormancy. Although the mutants displayed a faster radicle protrusion than the wild type, all of them germinated $100 \%$ after 3 days (Fig. 1a). This phenotype was confirmed with after-ripened seeds that had been stratified at $4^{\circ} \mathrm{C}$ for 3 days (Fig. $1 \mathrm{~b}$ and Supplementary Fig. 1). These observations indicated that SNL1 and SNL2 regulate seed germination independent of dormancy control. The enhanced germination of snllsnl2 was analysed in more detail by measuring radicle length after 2 days imbibition. In snllsnl2, $60 \%$ of the radicles were longer than $1,000 \mu \mathrm{m}$ and $40 \%$ in between 500 and $1,000 \mu \mathrm{m}$. These percentages were, respectively, 30 and $50 \%$ in Col (Fig. 1c), confirming the faster radicle growth in snllsnl2.

Auxin-related genes show enhanced expression in snl1snl2. We previously performed an RNA-sequencing (RNA-seq) analysis of $12 \mathrm{~h}$ imbibed freshly collected seeds of the snllsnl2 mutant and 

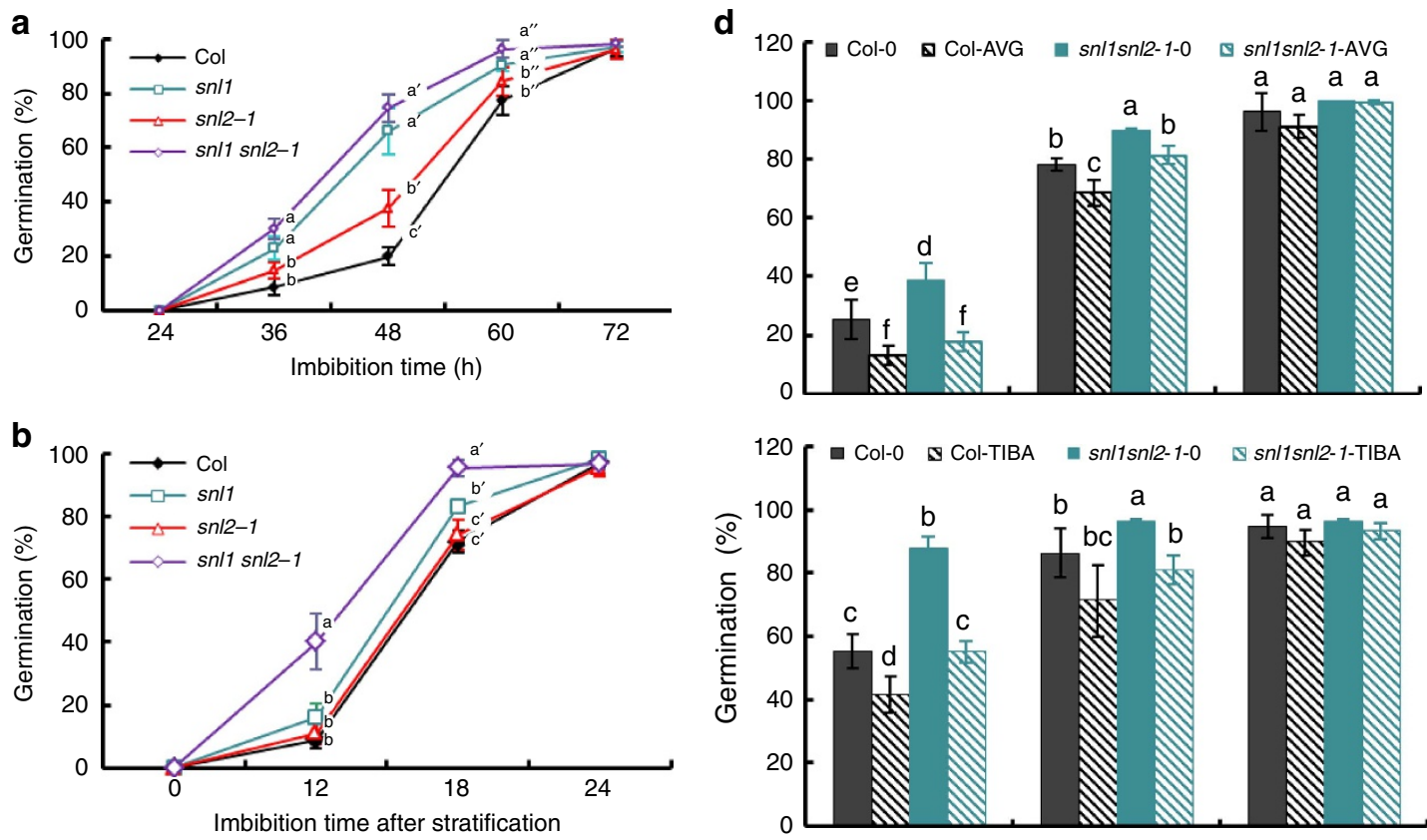

(h)
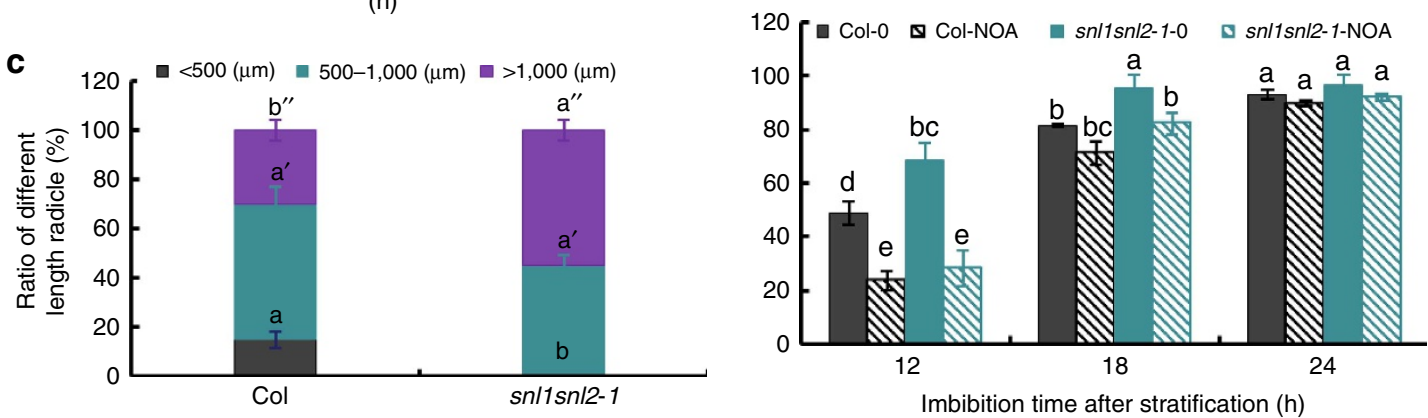

Figure 1 | Seed germination of $\mathbf{s n l}$ mutants and their response to auxin pathway inhibitors. (a,b) Germination phenotypes of wild-type Col, snl1, snl2-1 and snl1sn/2. Seeds were after-ripened for 4 months at room temperature before the germination experiment to complete dormancy release (a) or afterripened and subsequently stratified for 3 days at $4{ }^{\circ} \mathrm{C}(\mathbf{b})$. Percentages of seed germination are means ( \pm s.d.) based on the seeds from eight individual plants. Different letters at the same timepoint indicate a significant difference determined by Tukey's honestly significant difference (HSD) test $(P<0.05)$. (c) Radicle length after 2 days imbibition of after-ripened seeds from Col and sn/7sn/2-1. The radicles were divided in three groups according to their size. Data are means ( \pm s.e.) of two independent experiments with three technical replicates $(N=2, n=3 \times 30$ seeds). Different letters indicate a significant difference determined by Tukey's HSD test $(P<0.05)$. (d) Germination percentage of $\mathrm{Col}$ and sn/7sn/2 after exogenous application of auxin synthesis inhibitor AVG (top panel, $40 \mu \mathrm{M}$ ) and auxin transport inhibitors TIBA (middle panel, $25 \mu \mathrm{M}$ ) and NOA (bottom panel, $25 \mu \mathrm{M}$ ). The seeds were afterripened and stratified. Percentages of seed germination were shown after 12,18 and $24 \mathrm{~h}$ imbibition ( \pm s.d., $n=8$ ). Different letters indicate a significant difference determined by Tukey's HSD test $(P<0.05)$.

Col to identify genes with altered transcript levels ${ }^{32}$. A detailed analysis of these data showed that a high number of auxin-related genes had significantly increased transcript levels in the snllsnl2 mutant compared with wild-type Col (Supplementary Table 1). The role of auxin in the germination of snllsnl2 was analysed by imbibing seeds in the presence of the auxin synthesis inhibitor aminoethoxyvinylglycine (AVG) ${ }^{33}$ and the auxin transport inhibitors 2,3,5-triidobenzoid acid (TIBA) and 1-naphthoxyacetic acids (1-NOA). Interestingly, all three inhibitors caused a decrease in germination speed for both snl1snl2 double mutant and wild-type Col. In response to AVG, TIBA and NOA, the germination percentages were reduced from 25 to $13 \%$, from 55 to $41 \%$ and from 49 to $24 \%$ for Col, and from 39 to $17 \%$, from 88 to $55 \%$ and from 68 to $28 \%$ for snl 1 snl2, respectively, at $12 \mathrm{~h}$ after imbibition (Fig. 1d). In general, this inhibition was stronger for the snl1snl2 double mutant compared with wild type. Because a positive influence of auxin on seed germination has been reported ${ }^{16,17,19}$, we speculated that the accelerated seed germination of the $s n l$ mutants is caused by an enhanced auxin response.

Auxin synthesis and transport are enhanced in snl1snl2. Quantitative reverse transcription-PCR (qRT-PCR) was used to confirm genes associated with auxin synthesis, transport and signalling had increased transcript levels in $s n l$ mutant seeds. Almost all tested genes showed significantly increased transcript levels in after-ripened dry and $8 \mathrm{~h}$ imbibed snll and snl1snl2 seeds compared with wild-type Col (Fig. 2). However, after $24 \mathrm{~h}$ imbibition several of these genes showed similar transcript levels in $s n l$ mutants and wild type. In general, we observed a positive correlation between transcription levels of auxin pathway genes and seed germination speed in the snllsnl2 double mutant (Figs 1 and 2) ${ }^{17}$.

To further study the role of SNL1 and SNL2 in the auxin pathway during seed germination, the auxin-responsive reporter DR5::GUS was introduced into the $s n l$ mutants. The abundance of 

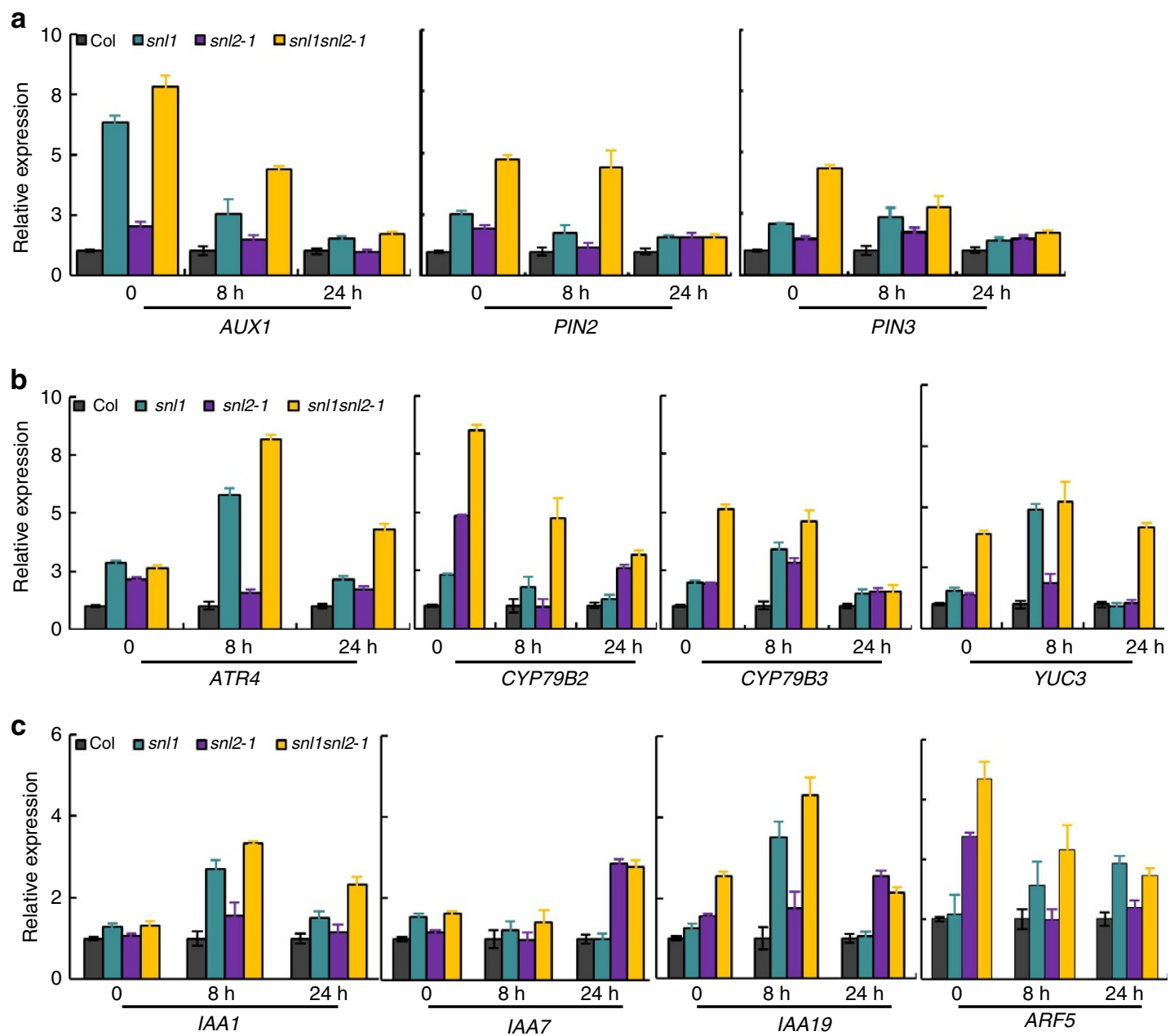

Figure 2 | The snl mutants show enhanced transcript levels of auxin-related genes during seed imbibition. The relative expression of (a) auxin transport-related genes, (b) auxin synthesis-related genes and (c) auxin signalling-related genes in seeds of Col, sn/1, sn/2 and sn/7snl2 measured by qRT-PCR. Each experiment had three biological repeats and the average value is shown with s.e. Transcript levels were normalized to the level of ACTIN8. The relative transcript level in Col is set as one. The seeds for RNA isolation were fully after-ripened and imbibed for 0,8 and $24 \mathrm{~h}$.

the GUS signal was analysed during seed germination and early seedling establishment. DR5::GUS signals in the embryo were significantly higher in snl1 and snllsnl2 compared with Col wild type and snl2 during radicle protrusion and early growth, but not in the mature seedling (Fig. 3a-f, and Supplementary Figs 2 and 3). In particular, DR5::GUS levels were increased in the radicle tip of snl1snl2 during seed imbibition before radicle protrusion (Fig. 3g,h). Quantification of the GUS signal by ImageJ software confirmed these observations (Fig. 3i). Moreover, quantification of IAA levels using gas chromatography-mass spectrometry (GC-MS) showed significantly higher levels in the seeds of snll and snl1snl2 compared with Col (Fig. 3j). Overall, the results indicated that SNL1 and SNL2 negatively regulate auxin levels during seed germination, which might affect germination speed.

Auxin promotes seed germination and activates cell cycling. To study the role of auxin during seed germination, fully after-ripened Col seeds were imbibed in the presence of low or high concentrations of IAA or the auxin analogue 2,4-dichlorophenoxyacetic acid (2,4-D). Both IAA and 2,4-D promoted germination speed at low doses $(0.05-5 \mathrm{nM})$ in stratified and non-stratified seeds (Fig. 4a,b and Supplementary Fig. 4a), and decreased germination speed at high doses $(0.05-5 \mu \mathrm{m})$ (Supplementary Fig. 4b,c). Imbibition of seeds in
$5 \mathrm{nM}$ IAA increased the DR5::GUS signal in the radicle tip (Supplementary Fig. 5), which implied that external IAA treatment enhanced the internal auxin response level.

Auxin can regulate plant development by inducing cell elongation and division ${ }^{34,35}$. Radicle elongation of Arabidopsis seeds does not require cell division, but cell elongation of the hypocotyl-radicle transition zone. The increase of cell size in the region of elongation is accompanied by an increase in the proportion of $4 \mathrm{C}$ and endopolyploid (8C and 16C) nuclei ${ }^{36}$. We analysed the DNA content of embryo cells by flow cytometry and detected enhanced levels of $4 \mathrm{C}$ and $8 \mathrm{C}$ nuclei in 24 and $36 \mathrm{~h}$ imbibed seeds in the presence of $5 \mathrm{nM}$ IAA or 2,4-D (Fig. 4c). These results suggest that auxin may promote germination speed by stimulating cell mitosis or endocycle.

AUX1 plays a key role in seed germination mediated by SNLs. Auxin is likely to be important for the increased germination speed of the snl1snl2 double mutant (Figs 1-3). We next aimed to identify key factors in the auxin pathway involved in the regulation of germination speed downstream of SNL1 and SNL2. Mutants of auxin-related genes (for example, PIN2, PIN3, CYP79B2, CYP79B3 and YUC3) that showed enhanced transcript levels in snl1snl2 (Supplementary Table 1) did not show obvious seed germination phenotypes. However, the aux1-21 and aux1-22 


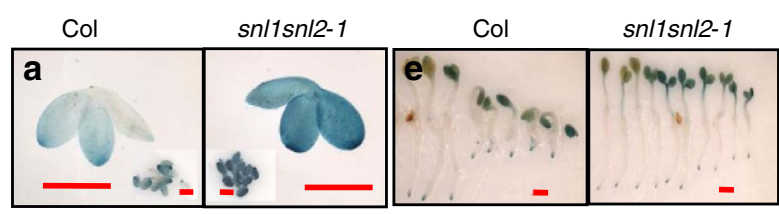

Imbibition $2 \mathrm{~h}$

mbibition $72 \mathrm{~h}$ (seedlings)
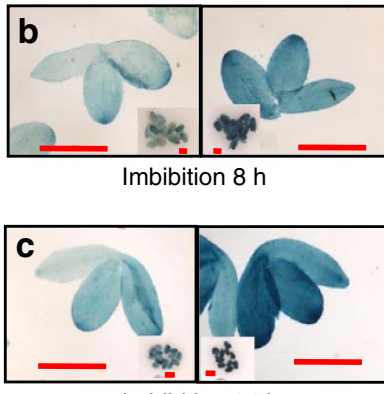

Imbibition $24 \mathrm{~h}$

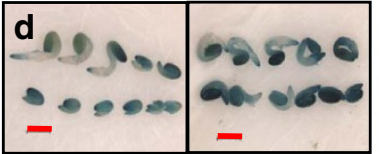

Imbibition $48 \mathrm{~h}$

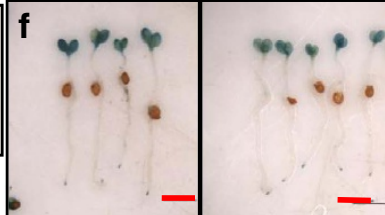

Imbibition 5 days (seedlings)

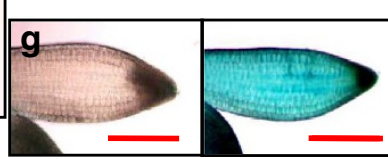

Imbibition $0.5 \mathrm{~h}$

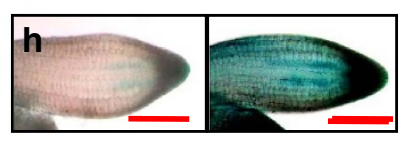

Imbibition $24 \mathrm{~h}$ i
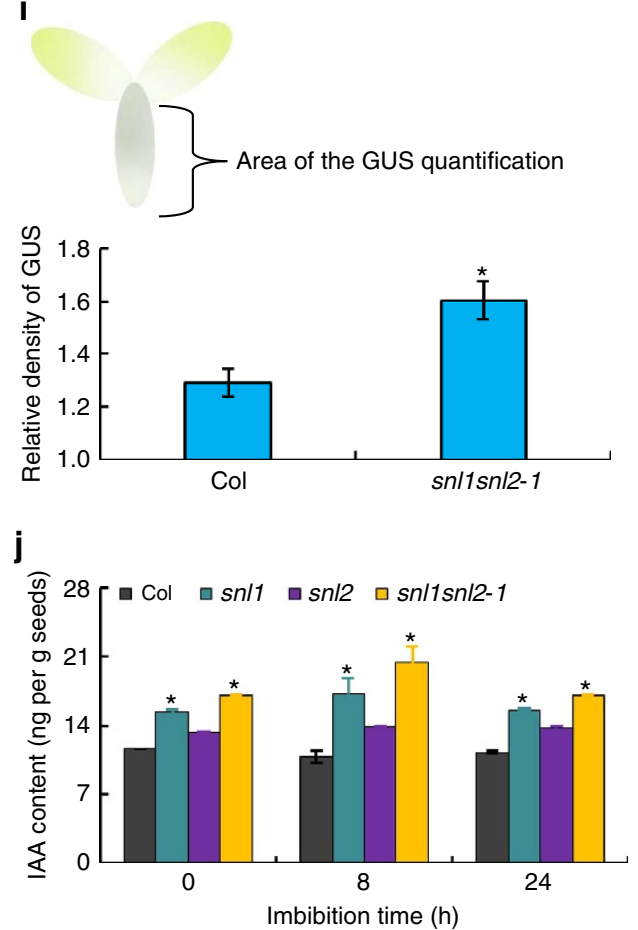

Figure 3 | The sn/1sn/2 mutant accumulates high levels of auxin. (a-h) Signals of the auxin reporter DR5::GUS were visualized by GUS assay and imaged in embryos and seedlings after $0.5 \mathrm{~h}-5$-day imbibition of Col and snl1sn/2 seeds. Scale bars, $500 \mu \mathrm{m}$ (a-f); $200 \mu \mathrm{m}$ (g,h). (i) The GUS intensity in the radicle of Col and snl1sn/2 was measured by ImageJ software, the background was set as one and relative levels are shown. Error bars denote s.d. ( $n>30$ ).

The radicle area used for GUS quantification is indicated in the upper diagram. The asterisk indicates a significant difference according to Student's $t$-test $(P<0.05)$. (j) The IAA content in dry, 8 and $24 \mathrm{~h}$ imbibed seeds of Col and sn/7sn/2 was determined by GC-MS. Three independent experiments were performed and the average value is shown with s.d. The asterisk indicates a significant difference according to Student's $t$-test $(P<0.05)$.

mutants did show weakly decreased germination of fresh seeds compared with wild type (Supplementary Fig. 6a). This phenotype was not visible in after-ripened stratified seeds (Supplementary Fig. 6b), suggesting that AUX1 is a weak seed dormancy regulator. The aux1-T mutant, another mutant allele of AUX1 (ref. 37), behaved similarly (Supplementary Fig. 6b). RNA-seq data and qRT-PCR results showed that auxin-related genes were upregulated in snl1snl2 (Fig. 2 and Supplementary Table 1$)^{32}$. Therefore, we cloned AUX1, ARF5, ATR4, CYP79B2, YUC3 and so on (Supplementary Table 1) under the $12 \mathrm{~S}$ promoter to obtain high transcript levels in seeds. Transgenic plants containing these constructs were obtained and their seed germination phenotypes were analysed. After-ripened and stratified seeds of transgenic lines with 12Spro::AUX1 displayed significantly accelerated radicle protrusion (Fig. $5 \mathrm{a}-\mathrm{c}$ ). Interestingly, combining the aux1-21 mutant with the snl1snl2 double mutant restored the enhanced germination of snl1snl2 back to wild-type levels (Fig. 5d). This implied that AUX1 has an important role in the regulation of germination speed downstream of SNL1 and SNL2 (Fig. 5d). The transgenic lines that over-express other auxin-related genes did not show seed germination phenotypes.

Previous studies have shown that loss of AUX1 function resulted in decreased auxin levels ${ }^{37-39}$. Accordingly, we observed enhanced IAA levels in dry and imbibed seeds of AUX1 over-expression lines using GC-MS (Fig. 5e). Furthermore, introduction of the auxin reporter DR5::GUS into the 12Spro::AUX1 transgenic line showed enhanced GUS signals in the 12Spro::AUX1 radicle (Fig. 5f). These results suggest that AUX1 may be involved in radicle growth and seed germination by transporting auxin and modifying its accumulation in the radicle tip.
AUX1 accumulate in the radicle tip of snl1snl2. We further studied the role of AUX1 in the enhanced germination speed of snl1snl2 by introducing an AUX1pro::AUX1-YFP construct in this double mutant by crossing. The fluorescence signal from AUX1-YFP accumulated gradually during imbibition in both wild-type Col and snl1snl2 mutant background. However, the double mutant showed a higher intensity of fluorescence than the wild type (Fig. 6a). Immunoblot analysis also showed a higher abundance of the AUX1-YFP protein in 24 and $48 \mathrm{~h}$ imbibed snl1snl2 seeds compared with Col (Fig. 6b). This suggests that AUX1 has a role in the enhanced germination speed of the snl1snl2 double mutant.

AUX1 is a target of histone deacetylation mediated by SNLs SNL1 and SNL2 function in histone H3K9/K18 deacetylation ${ }^{32}$. Therefore, AUX1 could be a target of histone deacetylation mediated by SNL1 and SNL2. We analysed the acetylation levels of AUX1 in imbibed seeds of Col and snl1snl2 by ChIP-qRTPCR using H3K9K18ac antibody- and gene-specific primers (Supplementary Table 2). Enhanced acetylation levels were detected in the $\mathrm{C}$-terminal gene-coding region of AUX1 in snl1snl2 (Fig. 7a,b) supporting previous reports that AUX1 is a target of $\mathrm{H} 3 \mathrm{~K} 9$ acetylation ${ }^{40}$. Several additional genes were analysed, and the snllsnl2 double mutant showed increased acetylation at the promoter regions (region 1) of PIN2, and CYP79B2 (Supplementary Fig. 7) ${ }^{40}$. To verify the direct interaction between SNL1 and auxin-related genes during histone deacetylation, we constitutively expressed $3 \times$ FLAG-SNL1 in the snl1snl2 double mutant and obtained two homozygous lines, 1-6 and 2-6. qRT-PCR and seed germination assays showed that a wild-type SNL1 expression level and seed germination phenotype 
a

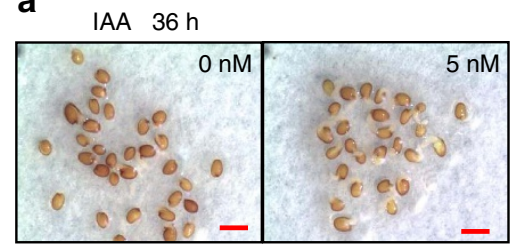

b

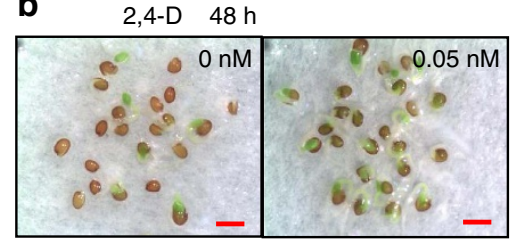

C

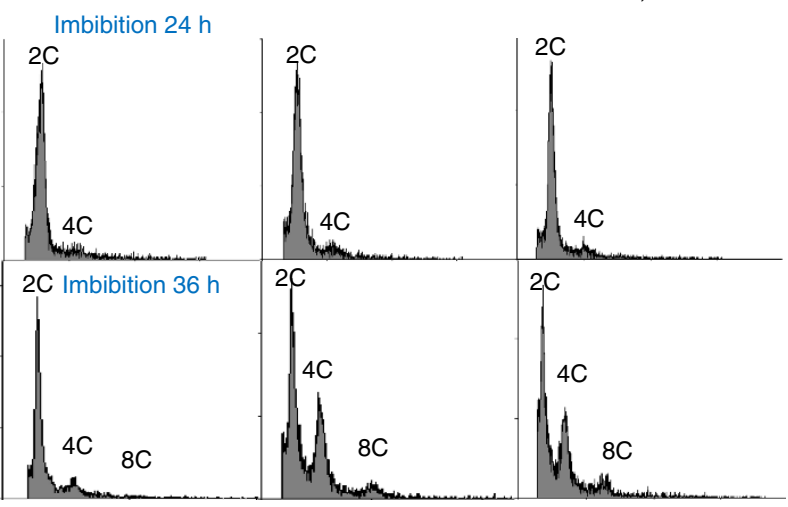

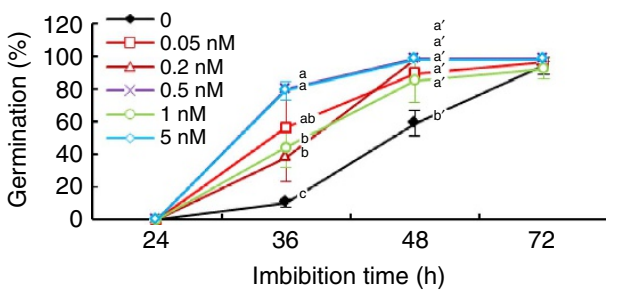

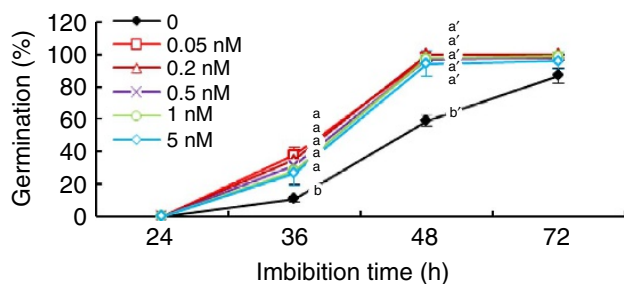

2,4-D

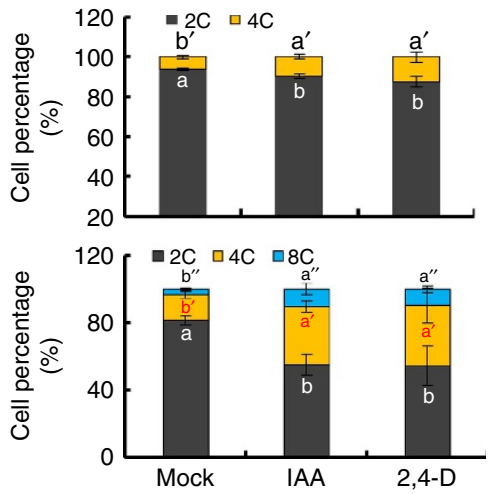

Figure 4 | Auxin treatment with low doses stimulates seed germination and enhances DNA content. (a,b) Germinating seeds of Col (left) are shown after $36 \mathrm{~h}$ imbibition in $5 \mathrm{nM}$ IAA or mock solution (a) and $48 \mathrm{~h}$ imbibition in $0.05 \mathrm{nM} 2,4-\mathrm{D}$ or mock solution (b). The graphs on the right show seed germination percentages during imbibition with different concentrations of (a) IAA and (b) 2,4-D (0.05-5 nM) treatment. Different letters at the same timepoint indicate a significant difference determined by Tukey's honestly significant difference (HSD) test $(P<0.05)$. Scale bars, $1 \mathrm{~mm}$. (c) Changes in nuclear ploidy levels during seed imbibition. Histograms representing DNA content of embryo cells during seed imbibition (left). Fully after-ripened wild-type Col seeds were imbibed for $24 \mathrm{~h}$ (upper panel) or $36 \mathrm{~h}$ (lower panel) in a mock solution, $5 \mathrm{nM}$ IAA or 0.05 nM 2,4-D. 2C peak, G1 DNA content; 4C peak, G2 DNA content; 8 C and 16C peaks, endoreduplicating cells. The graphs in the right show a quantification of the cell numbers at the different stages of the cell cycle based on the histograms. The bars indicate s.d. of three biological replicates and different letters indicate a significant difference determined by Tukey's HSD test $(P<0.05)$.

were recovered in the two transgenic lines (Supplementary Fig. 8). A ChIP-qRT-PCR analysis using anti-FLAG antibody indicated that $3 \times$ FLAG-SNL1 proteins were mainly located in the $C$ terminus of the $A U X 1$ gene (Fig. 7c), indicating that a potential interaction between SNL1 and AUX1 occurred in the C terminus of the gene-coding region (Fig. $7 \mathrm{c}$ ). Moreover, $3 \times$ FLAG-SNL1 enrichment was also detected in the promoter region (region 1 ) of PIN2, and the promoter (region 1) and C-terminal gene-coding region (region 3) of CYP79B2, which is similar to the results from the H3K9K18ac ChIP assay (Supplementary Fig. 7). These data suggest that the upregulation of $A U X 1$ and other auxin-related genes in snllsnl2 are tightly associated with increased histone H3K9K18 acetylation resulting from the loss of SNL1 and SNL2 function.

CYCDs are regulated by AUX1 and SNLs. AUX1 localizes in the radicle tip of mature embryos ${ }^{38,41}$ and affects cell proliferation during embryogenesis in Arabidopsis ${ }^{23}$. To study the role of AUX1 in the promotion of radicle protrusion and growth, we counted the number of cells at the radicle tip in $24 \mathrm{~h}$ imbibed after-ripened seeds of Col, 12Spro::AUX1 and snl1snl2. We observed a higher number of radicle cells in 12Spro::AUX1 and snllsnl2 compared with Col wild type (Fig. 8a). A flow cytometric analysis indicated enhanced levels of $4 \mathrm{C}$ and $8 \mathrm{C}$ nuclei in 12Spro::AUX1 and snl1snl2 24 and $36 \mathrm{~h}$ imbibed seeds compared with Col (Fig. 8b). This indicated faster cell cycling, which could contribute to the faster radicle protrusion of 12Spro::AUX1 and snl1snl2 (Figs 1a and 5a). Cyclin D-type (CYCD) 1;1 and CYCD4;1 are two key genes involved in cell cycling and seed germination $^{42}$. Both of these genes showed enhanced transcript levels during imbibition of snl1snl2 and 12Spro::AUX1 compared with Col wild type (Fig. 8c,d). It has been reported that cyclins could be regulated by phytohormones ${ }^{43}$. Our experiments indicated that a low auxin dose induced the expression of CYCD1;1 and CYCD4;1, especially at early stages of imbibition (Supplementary Fig. 9). Moreover, loss of CYCD4;1 function in the 12Spro::AUX1 background reduced the germination speed of 12Spro::AUX1 back to the wild-type level. Similarly, knockout of CYCD4;1 in the snl1snl2 mutant restored seed germination speed to wild type (Fig. 8e). These results suggest that CYCD1;1 and $C Y C D 4 ; 1$ play an important role in radicle promotion and growth downstream of AUX1 and SNL1/SNL2. 
a

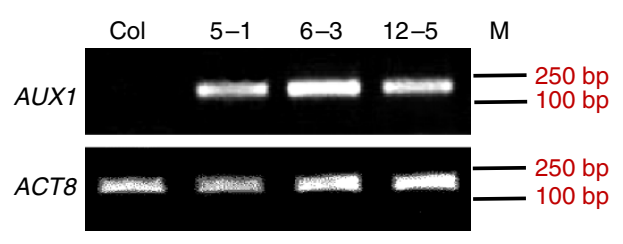

C

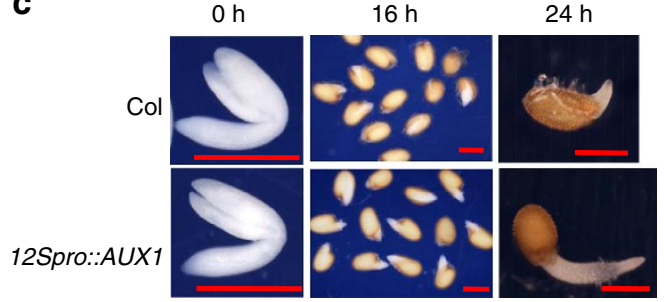

d

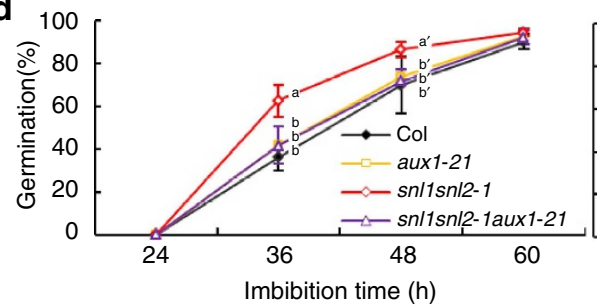

e

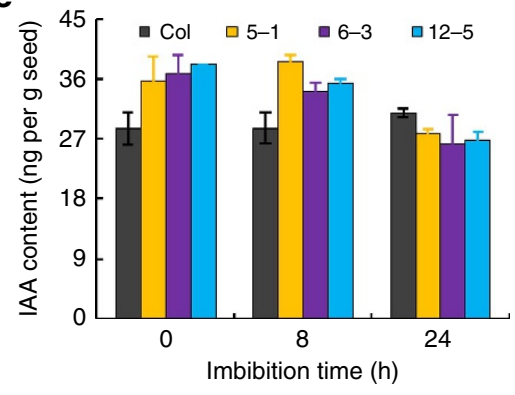

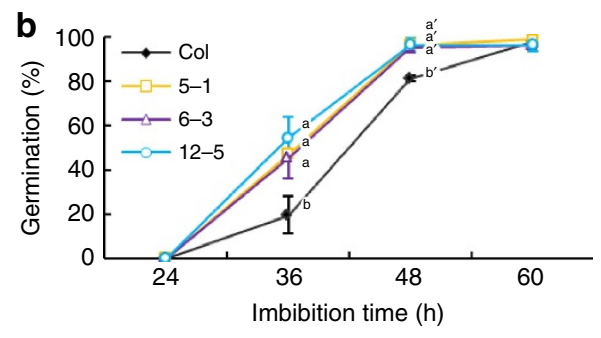

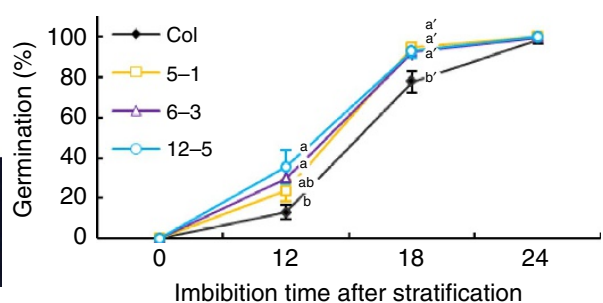

(h)

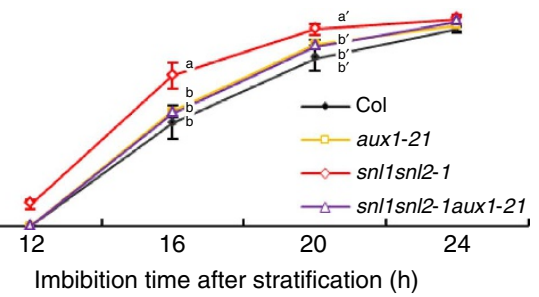

f

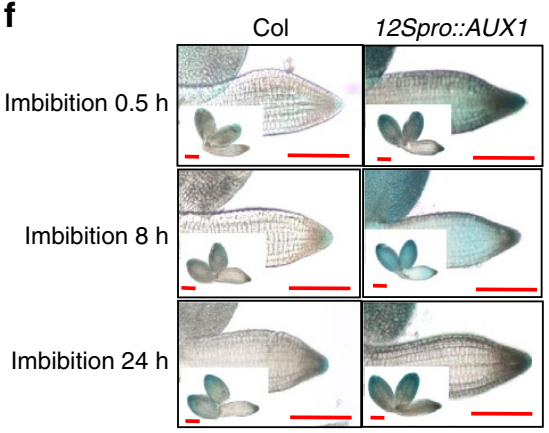

Figure 5 | Overexpression of AUX1 causes increased auxin level and seed germination. (a) AUX1 transcript levels in dry seeds of three independent homozygous transgenic lines containing 12Spro::AUX1 (5-1, 6-3 and 12-5). Semi-quantitative PCR was used to detect AUX1 transcript levels and the ACT8 gene was used as control. M, DNA molecular weight marker. (b) Seed germination during imbibition of fully after-ripened seeds from Col and 125 Spo::AUX1 lines (5-1, 6-3 and 12-5). Percentages of seed germination are means ( \pm s.d.) based on eight individual plants. Different letters at the same timepoint indicate a significant difference determined by Tukey's honestly significant difference (HSD) test $(P<0.05)$. (c) Germination phenotype of after-ripened stratified seeds from wild-type Col and three 12Spro::AUX1 homozygous transgenic lines. The left panel shows the photos of embryos and seeds from Col and the 12Spro::AUX1 homozygous line 5-1 at different imbibition times. The right panel shows germination percentages during imbibition. More than eight individual plants were counted and the average value is shown with the s.d. Different letters at the same timepoint indicate a significant difference determined by Tukey's (HSD) test $(P<0.05)$. Scale bars, $500 \mu \mathrm{m}$. (d) Germination phenotypes of after-ripened seeds without (left) or with stratification (right) from wild-type, aux1-21, snl1snl2-1 and the triple mutant snl1snl2-1aux1-21. Percentages of seed germination are means ( \pm s.d.) based on eight individual plants. Different letters at the same timepoint indicate a significant difference determined by Tukey's HSD test $(P<0.05)$. (e) The IAA content in

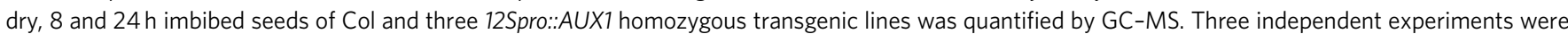
performed and the average value is shown with the s.d. (f) Photos showing GUS staining of radicles and embryos (inserts) from Col and a 12Spro::AUX1 transgenic line, both containing the auxin reporter gene DR5::GUS. Seeds were imbibed for $0.5,8$ and $24 \mathrm{~h}$. The experiment was performed twice with 15 embryos and representative photos are shown. Scale bars, $200 \mu \mathrm{m}$.

\section{Discussion}

SNL1 and SNL2 are highly expressed in dry seeds, but their transcript levels decrease during seed imbibition and germination (Supplementary Fig. 10) ${ }^{32}$ (http://bbc.botany.utoronto.ca/efp/ cgi-bin/efpWeb.cgi? primaryGene=AT3G01320\&modeInput $=$ Absolute). Here we showed that SNL1 and SNL2 negatively regulate radicle protrusion and growth during seed germination after dormancy release (Fig. 1a-c). SNL proteins are components of the histone deacetylation complex ${ }^{32}$. Interestingly, loss-offunction mutants of another component of this complex, HDA19, showed a similar accelerated seed germination phenotype as the snl1snl2 double mutant (Supplementary Fig. 11) suggesting that the SNL1/HDA19 complex controls seed germination via histone deacetylation.

The embryo plays an essential role in seed germination, both by activating its own growth potential and by transducing signals 


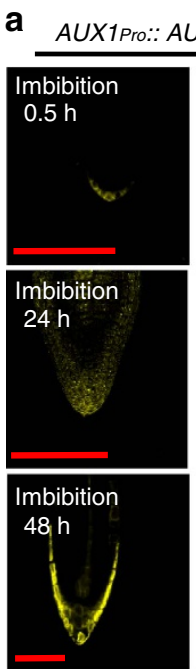

Fluorescense
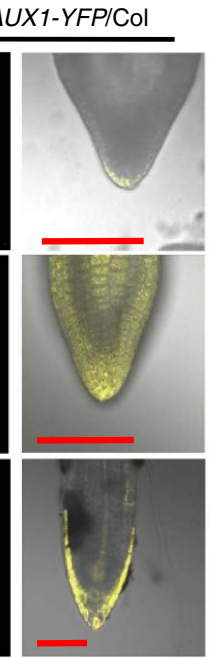

Merged

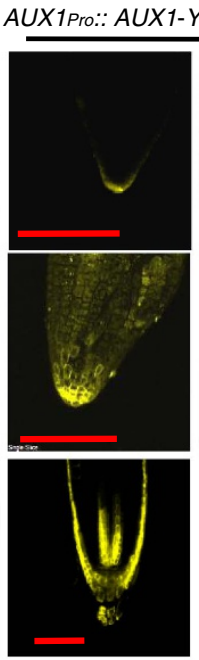

Fluorescense

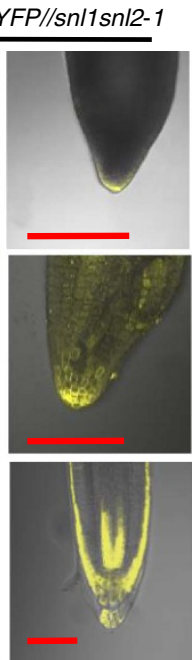

Merged

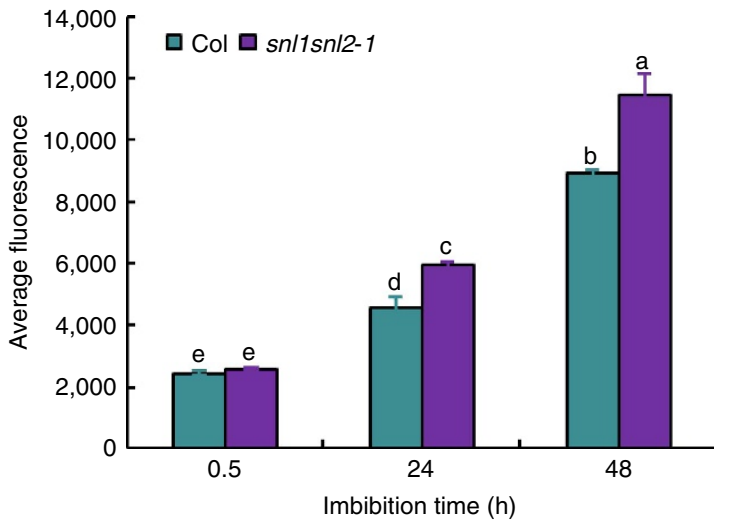

b

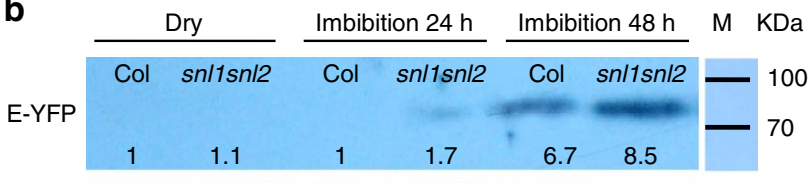

Loading

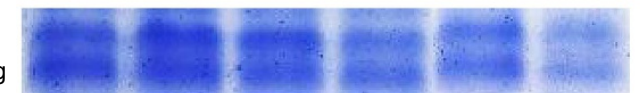

Figure 6 | AUX1 protein accumulates in the radicles of snl1sn/2 embryos.

(a) Upper panel, the AUX1-YFP protein was detected after 0.5, 24 and $48 \mathrm{~h}$ of seed imbibition in Col and snl1sn/2 radicles containing the AUX1pro::AUX1YFP construct. Higher-fluorescence signals were detected in the sn/1sn/2 mutant compared with the wild type. The lower panel shows a quantification of fluorescence signals by ImageJ software. The experiment was performed two times with independent plants, more than 15 embryos were observed and analysed statistically for each replicate and a representative result is shown. Different letters indicate a significant difference with Tukey's honestly significant difference test $(P<0.05)$. Scale bars, $200 \mu \mathrm{m}$. (b) Immunoblot analysis of AUX1-YFP protein accumulation in dry and imbibed seeds from $\mathrm{Col}$ and snl1sn/2 plants containing the AUX1pro::AUX1-YFP construct. The E-YFP monoclonal antibody was used for detection, and Coomassie blue staining was used as loading control. Numbers indicate relative AUX1-YFP protein levels, normalized to the loading control. The experiment was performed three times, similar results were obtained and a representative result is shown.

to weaken the endosperm. Activation of cell elongation is crucial for embryonic growth ${ }^{44,45}$. GAs have been suggested to play a key role in seed germination by increasing growth potential of the embryo and/or by weakening of the endosperm cap ${ }^{46-48}$.

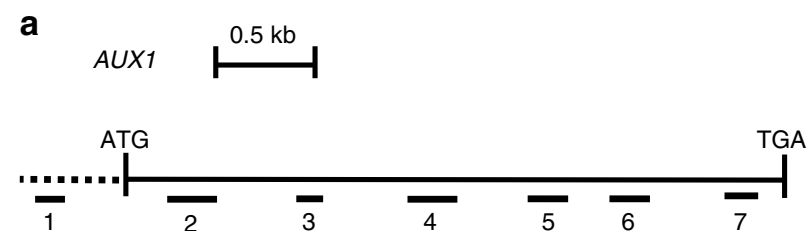

b

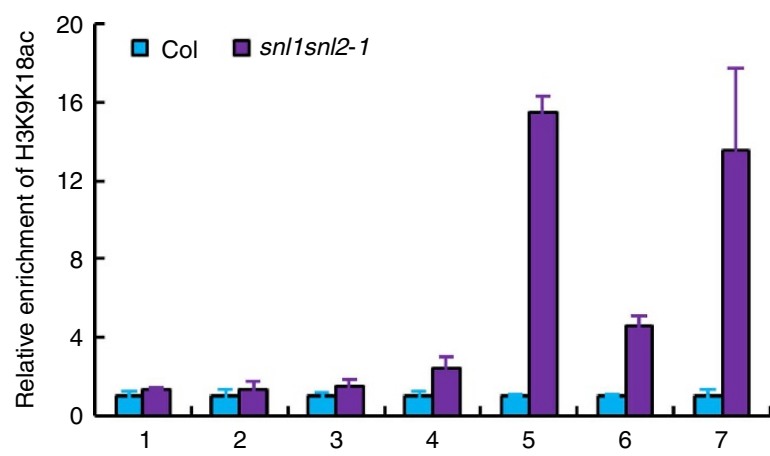

C

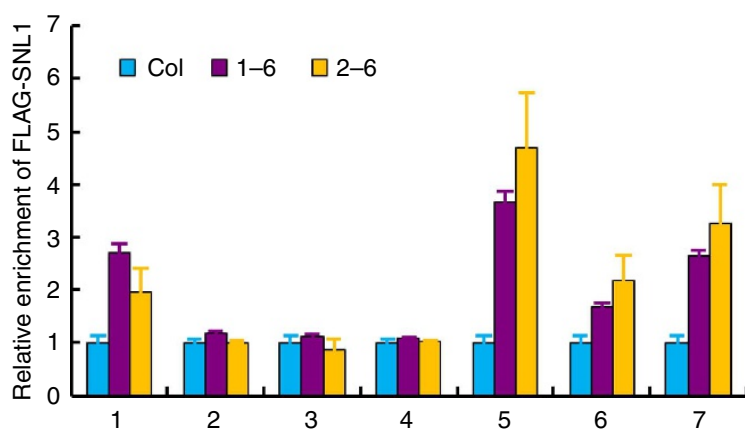

Figure 7 | The AUX1 gene has enhanced H3K9/K18 acetylation levels in snl1sn/2 seeds. (a) A schematic diagram of the AUX1 gene structure. The dashed line indicates the $\sim 500 \mathrm{bp}$ promoter sequence and the black line between the vertical dashes represents the open reading frame of the gene from start codon (ATG) to stop codon (TGA). The relative positions of the PCR-amplified fragments (1-7) for each tested region are depicted below the gene structure. Scale bar represents 500 bp. (b) ChIP analysis of H3K9/ K18 acetylation levels on AUX1. Immunoprecipitates were obtained from $12 \mathrm{~h}$ imbibed seeds of Col and sn/7sn/2 using H3K9/K18ac antibody. The accumulation of PCR product after immunoprecipitation has been normalized to ACTIN8. The bars indicate s.e.'s of three biological replicates. The numbers on the $x$ axis represent the PCR-amplified sites described in a (c) ChIP analysis of $3 \times$ FLAG-SNL1 levels on AUX1. Immunoprecipitates were obtained from 7-day-old seedlings of Col and 35Spro::FLAG-SNL1/ sn/7sn/2 lines (1-6 and 2-6) using a FLAG antibody. Relative amounts of the PCR products were calculated and normalized to ACTIN8, and the value in Col was set as one. The bars indicate s.e.'s of three biological replicates.

However, our previous work showed that SNL1 and SNL2 did not influence seed germination through the GA pathway ${ }^{32}$. A phenotypic analysis of mutants related to ABA hydrolysis and ethylene pathways suggested that these processes do also not play major roles in SNL1/SNL2 control of seed germination (Supplementary Fig. 12) ${ }^{49}$ although they are important in the regulation of seed dormancy in snllsnl2 (refs 32,49). Auxin has been shown to promote seed germination at low doses (Fig. 4) ${ }^{16,17}$. Therefore, the higher IAA accumulation in dry and imbibed seeds of snllsnl2 (Fig. 3j) might explain the increase in germination rate. Pharmaceutical analyses further demonstrated that inhibitors of auxin synthesis and transport 
reduce seed germination rate of $s n l$ mutants to wild-type levels (Fig. 1d), supporting the role of auxin in seed germination. This suggests that auxin distribution controlled by transporters might have an important role in seed germination of snllsnl2.

SNL1 represses gene transcription by histone deacetyla$\operatorname{tion}^{32,50}$. The enhanced transcript levels of auxin-related genes in snllsnl2 probably result from increased histone acetylation abundance. The ChIP assay with anti-H3K9/18ac and anti-FLAG confirmed this positive relationship (Fig. 7 and Supplementary Fig. 7). We hypothesized that one or more of the auxin-related genes could play a key role in the control of seed germination by SNL1 and SNL2. An analysis of a number of mutants and a
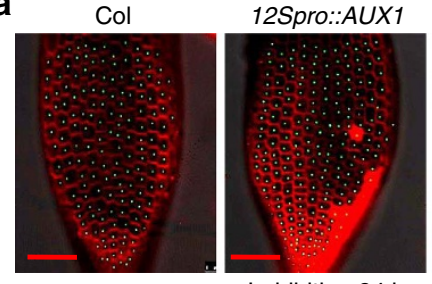

Imbibition $24 \mathrm{~h}$

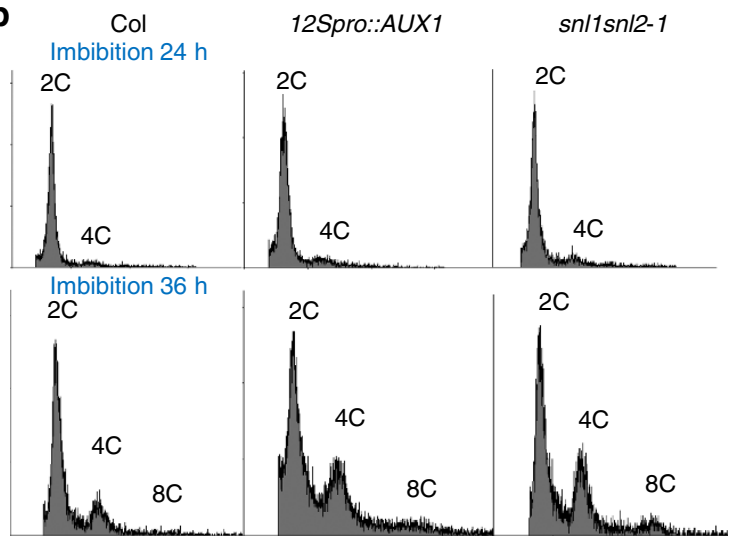

snl1snl2-1

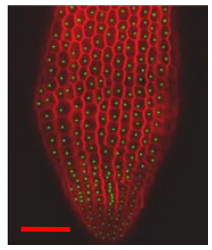

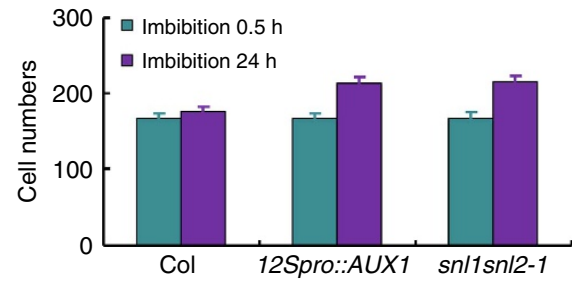

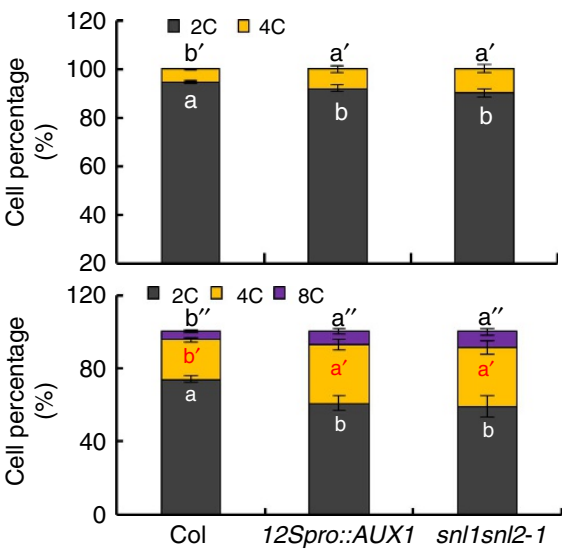

CYCD $4 ; 1$

C
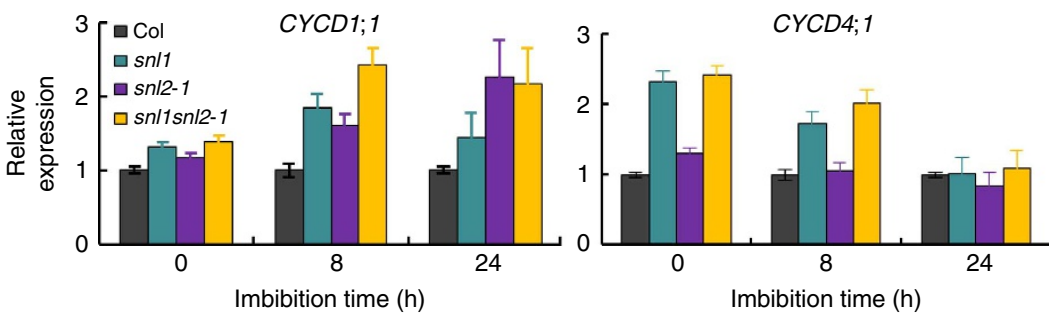

d
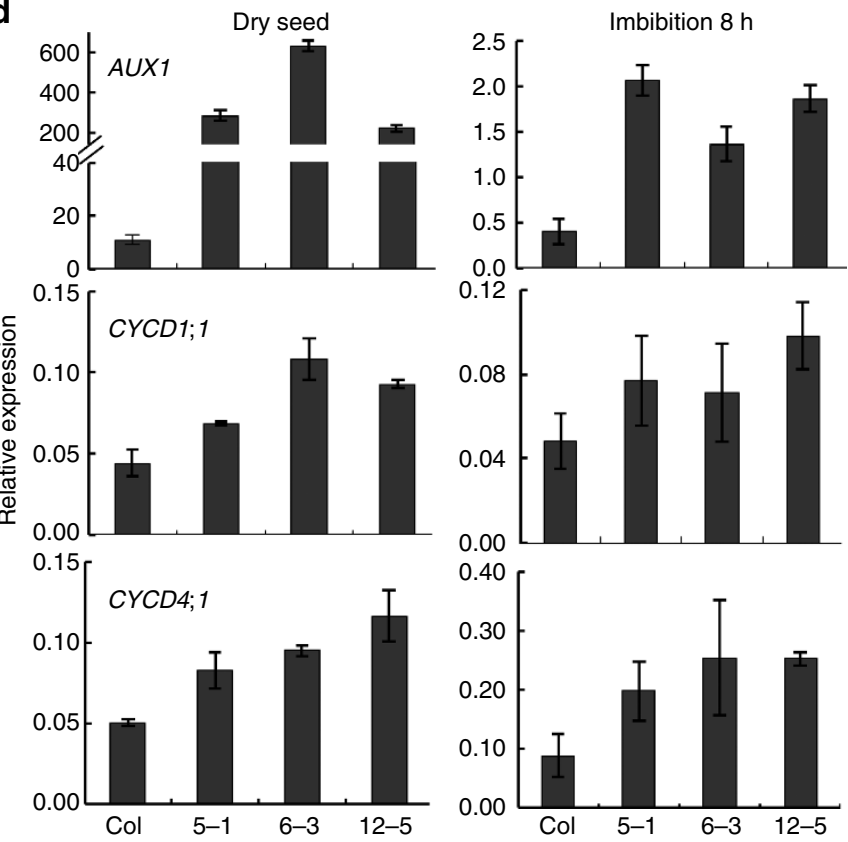

e
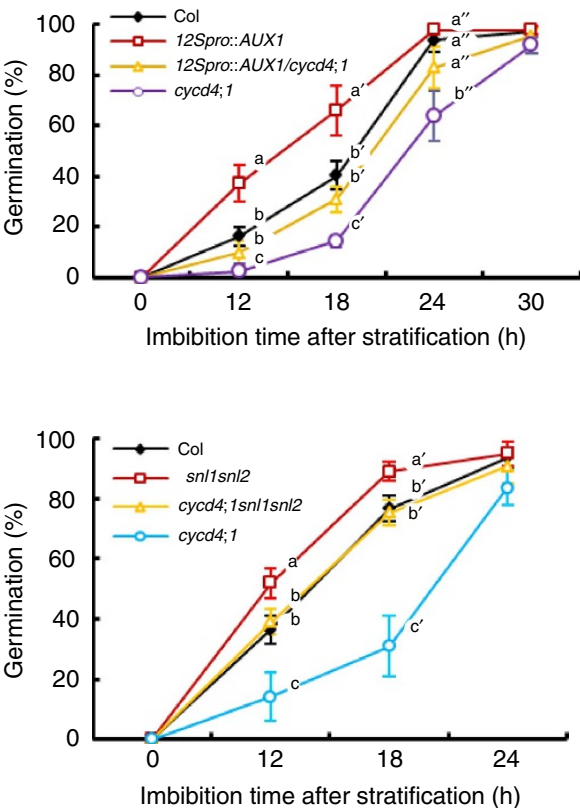
over-expression transgenic plants of several auxin-related genes, including AUX1, PIN2, PIN3 and ARF5 did not reveal seed germination phenotypes, except for $A U X 1$ (Fig. 5 and Supplementary Fig. 6). Moreover, loss of AUX1 function reduced the sensitivity of seed germination in response to exogenous auxin treatment at both low and high doses (Supplementary Figs 13 and 14). This suggests that AUX1 is a good candidate for the regulation of germination rate mediated by SNL1 and SNL2. Although loss of AUX1 function did not change germination speed (Supplementary Fig. 6b), AUX1 over-expression resulted in a significant acceleration in seed germination (Fig. 5a-c). Previous studies showed that AUX1 levels increase within the root tip during radicle growth on seed germination ${ }^{51}$, which is consistent with our results (Fig. 6a). Moreover, we found that AUX1 levels in the root tip of snl1snl2 are higher than that in the wild type during seed germination (Fig. 6). Genetic experiments showed that AUX1 is required for the accelerated germination speed of snl1snl2 since the snl1snl2auxl triple mutant had a similar germination speed as wild-type seeds (Fig. 5d). Band et $a l .{ }^{52}$ showed that the AUX1/LAX influx carriers are more important to create a proper distribution of auxin at the root tips of seedlings than efflux carriers such as PIN1 and PIN2. This is in agreement with our observations that germination speed depends more on AUX1 compared with the other auxin-related genes that we analysed. A possible explanation could be the more distinct functions of the $A U X 1 / L A X$ genes ${ }^{41}$ in plant development compared with the redundant functions of most auxin efflux transport genes ${ }^{53}$.

Loss of function of AUX1 causes altered auxin distribution and can result in decreased auxin content ${ }^{37-39}$. We propose that AUX1 regulates radicle protrusion and growth by modifying auxin distribution and concentration. In support, both GC-MS measurements and assays with the auxin reporter DR5::GUS showed increased auxin levels in dry and early imbibed seeds of 12Spro::AUX1 transgenic lines, but lower levels in the aux1-T mutant compared with the wild type (Fig. 5e,f and Supplementary Fig. 15). Altogether our results indicate that AUX1 has an important but not essential role in radicle protrusion and early growth by regulating auxin content and distribution in the radicle tip downstream of SNL1 and SNL2. However, the mechanisms by which auxin promotes seed germination are not well understood. Auxin is known to influence plant development by controlling cell division and proliferation ${ }^{34,35}$. Previous studies have shown that activation of cell division is important to promote successful seed germination and early seedling growth in Arabidopsis ${ }^{42}$. We investigated cell proliferation during seed germination of $s n l$ mutants and 12Spro::AUX1 transgenic lines, and obtained four pieces of evidence indicating that cell division within the radicle tip is important for their increased germination speed. First, the radicle tip contains more cells in 12Spro::AUX1 lines and snl1snl2 compared with wild type during seed imbibition (Fig. 8a). Second, a flow cytometric analysis showed that imbibed seeds of 12Spro::AUX1 and snl1snl2 contained a higher percentage of polyploid embryo cells compared with wild type (Fig. 8b). Third, the cell division-related genes CYCD1;1 and CYCD4;1, which encode D-type cyclins that act as rate limiting controllers of germination rate ${ }^{42}$, showed higher expression levels in the seeds of 12Spro::AUX1 and snllsnl2 compared with wild type (Fig. 8c,d). Fourth, loss of CYCD4;1 function in 12Spro::AUX1 or snllsnl2 background reduced seed germination speed to wild-type levels (Fig. 8e). These results indicated that the accelerated radicle protrusion and early growth of snl1snl2 and lines over-expressing AUX1 could be at least partially caused by a higher cell mitosis or endocycle mediated by the D-type cyclins CYCD1;1 and CYCD4;1. Previous reports indicated that some $C Y C D s$ can be induced by auxin ${ }^{43}$. In accordance, we showed that exogenous application of low doses of IAA and 2,4-D increased CYCD1;1 and CYCD4;1 transcript levels in seeds (Supplementary Fig. 9). The radicle tips of snl1snl2 and 12Spro::AUX1 lines accumulated relatively high levels of auxin compared with wild type as shown by a GC-MS assay and the auxin response reporter DR5::GUS (Figs 3 and 5e,f). This is likely to contribute to the enhanced expression of $C Y C D 1 ; 1$ and $C Y C D 4 ; 1$ transcripts in the radicles of both lines, causing accelerated radicle protrusion and growth.

In this work we showed the role of SNL1 and SNL2 in germination speed, whereas we previously described the pathway by which SNL1 and SNL2 control seed dormancy establishment ${ }^{32}$. By combining these regulatory functions, we propose a complete model for the control of seed dormancy and germination by histone deacetylation associated with SNL1 and SNL2 (Fig. 9). During the embryo development and seed maturation the expression of SNL1 and SNL2 increased gradually, which decreased the acetylation level of ABA hydrolysis genes (CYP707A1 and CYP707A2) and some ethylene pathway genes (ACO1 and ACO4). This led to a promotion of ABA signals and a repression of ethylene signals, contributing to seed dormancy establishment. During imbibition and germination of after-ripened seeds, the expression of SNL1 and SNL2 declined, which increased the acetylation of auxin pathway genes (for example, AUX1) and activated their transcription. Subsequently, auxin levels and signalling increased, causing an activation of cell mitosis or endocycle to promote radicle growth and seed germination. We conclude that the complexes associated with SNL1 and SNL2 are important hubs for the establishment of seed dormancy and the regulation of germination in Arabidopsis.

\footnotetext{
Figure 8 | AUX1 over-expressing lines and sn/1sn/2 mutant show increased cell proliferation and CYCDs expression in the radicle during germination. (a) Cell numbers in the radicle of $24 \mathrm{~h}$ imbibed seeds from Col, 12Spro::AUX1 and sn/7sn/2 were counted after FM4-64 (T-3166; Molecular Probes) staining and fluorescence microscopy imaging. The images in the left show stained radicles. The graphs in the right show endodermis cell numbers in Col, 12Spro::AUX1 and sn/1sn/2 with s.d. $(n>30)$. This experiment was performed two times with independent plants, similar results were obtained and a representative result is shown. Scale bars, $50 \mu \mathrm{m}$. (b) Changes in nuclear ploidy levels during seed imbibition. Histograms representing DNA content of embryo cells during seed imbibition. After-ripened seeds of Col, 12Spro::AUX1 and snl1sn/2 were imbibed for $24 \mathrm{~h}$ (upper panel) or $36 \mathrm{~h}$ (lower panel). 2C peak, G1 DNA content; 4C peak, G2 DNA content; 8C and 16C peaks, endoreduplicating cells. The graphs in the right show a quantification of the cell numbers at the different stages of the cell cycle based on the histograms. The bars indicate s.d. of three biological replicates and different letters indicate a significant difference determined by Tukey's honestly significant difference (HSD) test $(P<0.05)$. (c) The relative expression of CYCD1;1 and CYCD4;1 in imbibed fully after-ripened seeds from Col and snl single and double mutants measured by qRT-PCR. Transcript levels were normalized to the level of ACTIN8. The relative transcript level in wild-type Col is set as one. The bars indicate s.e.'s of three biological replicates. (d) The relative expression of AUX1, CYCD1;1 and CYCD4;1 in fully after-ripened dry and imbibed seeds from Col and three 12Spro::AUX1 transgenic lines measured by qRT-PCR. Transcript levels were normalized to the level of ACTIN8. The bars indicate s.e.'s of three biological replicates. (e) Germination phenotypes of 12Spro::AUX1/cycd4;1 (top) and the triple mutant $c y c d 4 ; 1 s n / 7 s n / 2-1$ (bottom). Seeds were stratified by imbibition for 3 days at $4{ }^{\circ} \mathrm{C}$ before germination. Percentages of seed germination are means ( \pm s.d.) based on the seeds from eight individual plants. Different letters at the same timepoint indicate a significant difference determined by Tukey's HSD test $(P<0.05)$.
} 


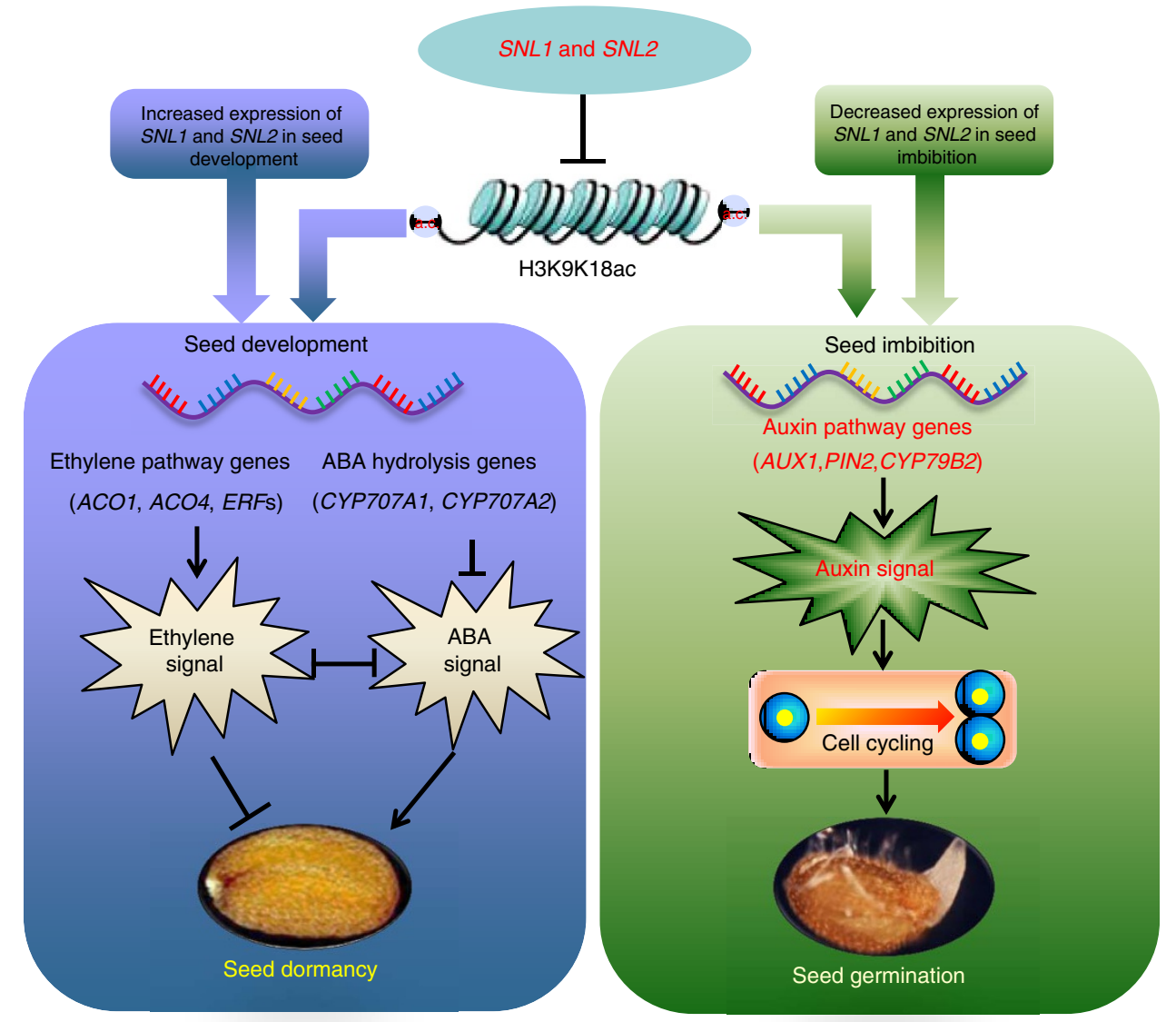

Figure 9 | A hypothetical model for the regulation of seed dormancy and germination by SNL1 and SNL2. The expression of SNL1 and SNL2 increases gradually during embryo development and seed maturation causing a decrease in acetylation level of ABA hydrolysis genes (CYP707A1 and CYP707A2) and some ethylene pathway genes (ACO1 and ACO4). This promotes ABA signalling and represses ethylene signalling leading to the establishment of seed dormancy. During imbibition of after-ripened seeds, the expression of SNL1 and SNL2 declines, causing an increase in acetylation levels of auxin pathway genes (for example, AUX1). Subsequent activation of their transcription leads to increased auxin levels and signalling, followed by enhanced cell division promoting seed germination.

\section{Methods}

Plant materials and growth conditions. All experiments were performed with Arabidopsis thaliana Columbia $(\mathrm{Col})$ wild-type plants, or mutants in the Col background. T-DNA insertion lines Sail_1151_F09 (snl1), Salk_097168 (snl2-1), Salk_073 549 (snl2-2) and GK_344D08 (cycd4; 1$)$ were previously described ${ }^{32,42}$, GK_900B12 (snl1-2) was ordered from NASC (University of Nottingham) and homozygous lines were identified by genome PCR using primers 900B12LP/RP and a T-DNA primer (Supplementary Table 2). Mutants of the auxin pathway genes, aux1-21, aux1-22, aux1-T, pin2 (eir1-1), pin3-3 and pin3-5 have been described before ${ }^{37,54,55}$. Other T-DNA mutants associated with the auxin pathway (for example, YUC3, CYP79B2 and CYP79B3) were ordered from ABRC (Ohio State University), identified by genome PCR and analysed for their seed germination phenotypes. DR5::GUS and AUX1pro::AUX1-YFP lines have been previously described ${ }^{56,57}$ and were individually crossed into snl1snl2. The double mutant snl1-2snl2-1 and the triple mutants snl1snl21 aux 1-21 and cycd4;1snl1snl2 were produced by crossing, as well as the 12Spro:: $A U X 1 / c y c d 4 ; 1$. Homozygous plants were isolated from F2 populations and F3 plants or further generations were used for analysis.

Seeds were sown in soil and grown in the greenhouse under photoperiodic cycles of $16 \mathrm{~h}$ light and $8 \mathrm{~h}$ dark at $22^{\circ} \mathrm{C}$. Seeds were sown on half-strength Murashige and Skoog (MS) medium after sterilization with $10 \%(\mathrm{v} / \mathrm{v}) \mathrm{NaClO}$. Plates were kept in the dark at $4{ }^{\circ} \mathrm{C}$ for 3 days to break dormancy (stratification) before moving into a climate chamber with a photoperiod of $16 \mathrm{~h}$ light and $8 \mathrm{~h}$ dark at $22^{\circ} \mathrm{C}$

Germination tests. Germination tests were performed as described by Alonso-Blanco et al. ${ }^{58}$ Radicle protrusion was regarded as seed germination completion. All germination experiments were performed on filter paper in $6 \mathrm{~cm}$ Petri dishes. Each germination experiment was performed with at least eight replicates (consisting of 80-100 seeds from one individual plant per Petri dish) per genotype. The average germination ratio was determined in a climate room $\left(25^{\circ} \mathrm{C}\right.$, $16 \mathrm{~h}$ light with $80-90 \mu \mathrm{mol} \mathrm{m}^{-2} \mathrm{~s}^{-1}$ light intensity). The radicle growth and seed germination speed were determined at different time points during imbibition (for example, 12, 18, 24 and $48 \mathrm{~h}$ ). For AVG (A6685; Sigma-Aldrich), TIBA (T5910; Sigma-Aldrich), NOA (255416; Sigma-Aldrich), IAA (I2886; SigmaAldrich) and 2,4-D (76514; Sigma-Aldrich) treatments, seeds were imbibed in Tris$\mathrm{HCl}(10 \mathrm{mM})$ buffer supplemented with or without (Mock: $10 \mathrm{mM}$ Tris- $\mathrm{HCl}$ buffer, $\mathrm{pH}$ 7.5) freshly prepared various concentrations of the reagents. Germination assays with seeds from different genotypes were performed with plants grown simultaneously in the same tray and stored under identical conditions. All the seed samples used were non-dormant and stored for at least 4 months at room temperature. For some experiments, seeds were given a 3 days stratification treatment at $4{ }^{\circ} \mathrm{C}$ before the germination test.

Constructs and plant transformation. Total RNA was isolated from young Col leaves using the Qiagen RNeasy kit (Qiagen). cDNAs were generated by SuperScript II reverse transcriptase (Invitrogen). The open reading frame of AUXI was amplified using Phusion DNA Polymerase (NEB) with primers AUX1-F/-R (Supplementary Table 2) and introduced into a Gateway Entry vector (Invitrogen). The Gateway Entry clones of ATR4, ARF5, CYP79B2 and YUC3 were ordered from NASC and destination clones were constructed by inserting cDNAs into the 12S::pLEELA vector, which is a derivative of pLEELA. The 12S::pLEELA vector was constructed by replacing the $35 \mathrm{~S}$ promoter with the $12 \mathrm{~S}$ promoter in pLEELA. CRUCIFERIN 3 (At4g28520) encodes a 12S seed storage protein and expresses specifically and highly in dry seeds ${ }^{59,60}$. The $12 \mathrm{~S}$ promoter $(1.85 \mathrm{~kb})$ was amplified with the primer pair $12 \mathrm{~S}-\mathrm{F}$ and $12 \mathrm{~S}-\mathrm{R}$ from the CRUCIFERIN 3 promoter region (Supplementary Table 2). The fusion genes were transformed in Col by 
Agrobacterium tumefaciens strain GV3101 pm90RK using the floral dip method ${ }^{61}$. Transformants were selected based on their ability to survive after 7 days in MS medium with $10 \mathrm{mgl}^{-1}$ DL-phosphinothricin. The 3:1 segregating transformant lines were selected on MS medium with $10 \mathrm{mgl}^{-1}$ DL-phosphinothricin. T3 homozygous transgenic plants were used for phenotypic analyses. The 35 Spro::3 $\times$ FLAG-SNL1 was constructed and transformed into the snl1snl2 mutant to obtain the complementing line 35Spro:: $3 \times$ FLAG-SNL1/snl1snl2-1. All of the constructs used in this study were confirmed by sequencing.

GUS staining and fluorescence visualization. Three independent transgenic lines were used for GUS staining using 5 -bromo-4-chloro-3-indolyl- $\beta$-D-glucuronic acid (X-Gluc, X8060; Amresco) as substrate ${ }^{62}$. Mature after-ripened embryos were soaked and imbibed in $\mathrm{dH}_{2} \mathrm{O}$ or IAA solution dissolved in Tris- $\mathrm{HCl}(10 \mathrm{mM})$ buffer for the indicated periods. Seed coats were removed under a dissecting microscope using a fine forceps and a $250 \mathrm{~mm}$ tungsten tip needle. Embryos dissected from the seed coat or seedlings were transferred to $1.5 \mathrm{ml}$ Eppendorf tubes containing GUS staining buffer. After staining, embryos and seedlings were soaked in 95 and $75 \%$ ethanol and thereafter examined and imaged under a stereoscope and Nikon 80i Upright microscope (Nikon) ${ }^{62}$. The radicle length was measured using the NIS-Elements D software and GUS density was analysed by ImageJ software ${ }^{63}$. Visualization of AUX1-YFP was conducted using a Zeiss confocal microscope (LSM 510 META) (excitation wavelength $480 \pm 20 \mathrm{~nm}$, emission wavelength $510 \pm 20 \mathrm{~nm}$ ). Zeiss LSM Image Browser software (version 3.2.0.70) was used for image acquisition.

Radicle cell number statistics. The dissected embryos were stained in FM4-64 (T-3166; Molecular Probes) at a final concentration of $10 \mu \mathrm{m}$ to visualize the cells with a Zeiss confocal microscope (LSM 510 META). The part from the tip to the middle of the radicle ( $1 / 2$ radicle length) was used for cell counting and statistically analysed. Only endodermis cells were captured and counted since the endodermis cells in the radicle tip differentiated earlier and stained better than other cells, which facilitated fluorescence signal observation and accurate statistics. Green spots were artificially added to the photo in cells to enable precise counting using Photoshop software. For each line, at least 30 embryos were stained and statistically analysed.

Protein extraction and immunoblotting. A unit of $20 \mathrm{mg}$ dry or imbibed seeds were ground in a mortar with liquid nitrogen, after which protein was extracted with a buffer containing $6 \mathrm{M}$ urea, $2 \mathrm{M}$ thiourea, $0.2 \%(\mathrm{v} / \mathrm{v})$ Triton X-100, $0.2 \%(\mathrm{w} / \mathrm{v})$ sarcosyl and $2 \mathrm{mM}$ dithiothreitol in $100 \mathrm{mM}$ Tris-Cl, pH 7.5. After two cycles of 30 min shaking the supernatant was collected by centrifugation at $4{ }^{\circ} \mathrm{C}$ for use ${ }^{64}$. Protein concentration was determined by Bradford dye reagent (Bio-Rad) using BSA as a standard. A unit of $50 \mu \mathrm{g}$ proteins were separated by electrophoresis on a $4-10 \%$ polyacrylamide gel and transferred to Immobilon-P membrane (Amersham) for immunoblotting. Traditional semi-dry transfer was performed using the TE77XP blot module (Hoefer). Protein accumulation was detected using anti-E-YFP monoclonal antibody (1: 1,000 dilutions; AB124-01, Toyephon) and visualized with X-film using a horseradish peroxidase-conjugated anti-mouse IgG secondary antibody (1: 5,000 dilutions; 330, MBL) and the Super ECL plus detection system (Applygen). The relative signal density was analysed by ImageJ software, the signal in dry Col seeds was set as one. Uncropped scans of all western blots and DNA gels are shown in Supplementary Fig. 16.

RNA extraction and qRT-PCR. Total RNA from seedlings or seeds was extracted using the Qiagen RNeasy kit and RNAqueous small-scale phenol-free total RNA isolation kit (Ambion) according to the manufacturer's instructions and reverse transcribed using the SuperScript RT-PCR system (Invitrogen). One microgram of total RNA was used for reverse transcription and semi-quantitative PCR, which was performed at least four times for each sample using rTaq polymerase (TaKaRa). ACTIN8 (ACT8) was amplified as a control. The linear range of detection for each transcript was monitored and samples were run with 35 cycles for AUX1 using primer pair AUX1-QF and AUX1-QR, and 30 cycles for ACT8. The qRT-PCR was performed using the KAPA SYBR FAST qPCR Kits (KAPA). The expression value for each gene was quantified using a standard curve with a serial dilution of plasmid of known concentration and normalized to the value of ACT8 (ref. 65). At least three biological replicates were analysed. Primers are listed in Supplementary Table 2. Mean values and standard errors were calculated from three biological replicates.

Measurement of free IAA. After-ripened seeds of Col, snl1, snl2-1, snllsnl2 mutants and 12Spro::AUX1 transgenic plants were used for the measurement of free IAA levels. The extraction, purification of samples and analysis of free IAA by GC-MS were performed according to the method described by Edlund et al. ${ }^{66}$, except that an Agilent 7000B GC-MS Triple Quad GC-MS was used with the separation performed in a DB-5ht column (Agilent). The internal standard $\left[{ }^{13} \mathrm{C}_{6}\right]$ IAA was purchased from Cambridge Isotope Laboratories (http://www.isotope.com).

ChIP assay. About $1.5 \mathrm{~g} 12 \mathrm{~h}$ imbibed seeds or 7-day-old seedlings were used for ChIP assay. Chromatin preparation and immunoprecipitation were performed as described ${ }^{67}$. Imbibed seeds or seedlings were fixed in $1 \%$ formaldehyde for $10 \mathrm{~min}$ in a vacuum. Glycine was added to a final concentration of $0.125 \mathrm{M}$, and the reaction was terminated by incubation for $5 \mathrm{~min}$ in a vacuum. Seedlings were rinsed three times with distilled water and frozen in liquid nitrogen. After isolation, chromatin was sheared to $500-2,000$ bp fragments by sonication (Branson Sonifier 250). Immunoprecipitation was performed by adding specific antibody and protein $\mathrm{G}$ agarose/salmon sperm DNA (Millipore) to the extract. ChIP assays were performed using $4 \mu \mathrm{g}$ anti-H3K9/18ac (07-593; Upstate) and anti-FLAG M2 (F1804; Sigma-Aldrich) antibodies. After washing, immune complexes were eluted from the protein $\mathrm{G}$ beads and reverse crosslinked by incubation at $65^{\circ} \mathrm{C}$ over night Samples were treated with proteinase $\mathrm{K}$ for $1 \mathrm{~h}$ at $65^{\circ} \mathrm{C}$ and RNase A for $1 \mathrm{~h}$ at $37^{\circ} \mathrm{C}$. DNA was extracted in a final volume of $40 \mu \mathrm{l}$ using the QIAquick PCR purification kit (Qiagen). A volume of $0.5 \mu \mathrm{l}$ DNA was used for each qRT-PCR. The qRT-PCR with SYBR Premix Ex Taq (TaKaRa) was carried out with a realtime system (Eppendorf). Each sample was assayed in triplicate by PCR. ACT8 was used as internal control in the ChIP assay ${ }^{32,65}$. Primers used for ChIP assays are listed in Supplementary Table 2 online.

Flow cytometry. Seed samples were collected at different stages of germination and more than 150 mature embryos were isolated from their seed coats. Nuclei were released by chopping and analysed with the Moflo XDP Cell Sorter (Beckman-Coulter) as described ${ }^{68}$. The data were analysed by Summit software and statistical significance analyses were made.

Statistical analysis. For physiological and biochemical data, an analysis of variance was performed to investigate whether there was a significant difference between the samples. If a significant difference was found, a Tukey's honestly significant difference test was performed to determine which samples were responsible for the significant differences.

Data availability. The authors declare that all data supporting the findings of this study are available within the paper and its Supplementary Information files or are available from the corresponding author on request.

\section{References}

1. Bewley, J. D. Seed germination and dormancy. Plant Cell 9, 1055-1066 (1997)

2. Bassel, G. W. et al. Genome-wide network model capturing seed germination reveals coordinated regulation of plant cellular phase transitions. Proc. Natl Acad. Sci. USA 108, 9709-9714 (2011).

3. Chen, H. et al. Integration of light and abscisic acid signaling during seed germination and early seedling development. Proc. Natl Acad. Sci. USA 105, 4495-4500 (2008).

4. Dekkers, B. J. et al. Transcriptional dynamics of two seed compartments with opposing roles in Arabidopsis seed germination. Plant Physiol. 163, 205-215 (2013).

5. Holdsworth, M. J., Bentsink, L. \& Soppe, W. J. Molecular networks regulating Arabidopsis seed maturation, after-ripening, dormancy and germination. New Phytol. 179, 33-54 (2008).

6. Finkelstein, R., Reeves, W., Ariizumi, T. \& Steber, C. Molecular aspects of seed dormancy. Annu. Rev. Plant Biol. 59, 387-415 (2008).

7. Koornneef, M., Bentsink, L. \& Hilhorst, H. Seed dormancy and germination. Curr. Opin. Plant Biol. 5, 33-36 (2002).

8. Finkelstein, R. R. \& Lynch, T. J. The Arabidopsis abscisic acid response gene ABI5 encodes a basic leucine zipper transcription factor. Plant Cell 12, 599-609 (2000).

9. Finkelstein, R. R. \& Somerville, C. R. Three classes of abscisic acid (ABA)Insensitive mutations of Arabidopsis define genes that control overlapping subsets of aba responses. Plant Physiol. 94, 1172-1179 (1990).

10. Koornneef, M. \& Van der Veen, J. H. Induction and analysis of gibberrellinsensitive mutants in Arabidopsis thaliana (L.) Heynh. Theor. Appl. Genet. 58, 257-263 (1980).

11. Sun, T., Goodman, H. M. \& Ausubel, F. M. Cloning the Arabidopsis GA1 locus by genomic subtraction. Plant Cell 4, 119-128 (1992).

12. Penfield, S., Gilday, A. D., Halliday, K. J. \& Graham, I. A. DELLA-mediated cotyledon expansion breaks coat-imposed seed dormancy. Curr. Biol. 16, 2366-2370 (2006).

13. Rajjou, L. et al. Seed germination and vigor. Annu. Rev. Plant Biol. 63, 507-533 (2012).

14. Brady, S. M., Sarkar, S. F., Bonetta, D. \& McCourt, P. The ABSCISIC ACID INSENSITIVE 3 ( $A B I 3)$ gene is modulated by farnesylation and is involved in auxin signaling and lateral root development in Arabidopsis. Plant J. 34, 67-75 (2003).

15. Liu, P. P. et al. Repression of AUXIN RESPONSE FACTOR10 by microRNA160 is critical for seed germination and post-germination stages. Plant J. 52, 133-146 (2007). 
16. He, J. et al. DEXH box RNA helicase-mediated mitochondrial reactive oxygen species production in Arabidopsis mediates crosstalk between abscisic acid and auxin signaling. Plant Cell 24, 1815-1833 (2012).

17. Hsueh, Y. L. \& Lou, C. H. Effects of 2,4-D on Seed germination and respiration. Science 105, 283-285 (1947).

18. Liu, X. et al. Auxin controls seed dormancy through stimulation of abscisic acid signaling by inducing ARF-mediated ABI3 activation in Arabidopsis. Proc. Natl Acad. Sci. USA 110, 15485-15490 (2013).

19. Wang, L. et al. Auxin response factor2 (ARF2) and its regulated homeodomain gene HB33 mediate abscisic acid response in Arabidopsis. PLoS Genet. 7, e1002172 (2011).

20. Ogawa, M. et al. Gibberellin biosynthesis and response during Arabidopsis seed germination. Plant Cell 15, 1591-1604 (2003).

21. Carrera, E. et al. Gene expression profiling reveals defined functions of the ATP-binding cassette transporter COMATOSE late in phase II of germination. Plant Physiol. 143, 1669-1679 (2007).

22. Thole, J. M., Beisner, E. R., Liu, J., Venkova, S. V. \& Strader, L. C. Abscisic acid regulates root elongation through the activities of auxin and ethylene in Arabidopsis thaliana. G3 (Bethesda) 4, 1259-1274 (2014).

23. Ugartechea-Chirino, Y. et al. The AUX1 LAX family of auxin influx carriers is required for the establishment of embryonic root cell organization in Arabidopsis thaliana. Ann. Bot. 105, 277-289 (2010).

24. Henderson, J. T. et al. PICKLE acts throughout the plant to repress expression of embryonic traits and may play a role in gibberellin-dependent responses. Plant Physiol. 134, 995-1005 (2004).

25. Perruc, E., Kinoshita, N. \& Lopez-Molina, L. The role of chromatin-remodeling factor PKL in balancing osmotic stress responses during Arabidopsis seed germination. Plant J. 52, 927-936 (2007).

26. Bouyer, D. et al. Polycomb repressive complex 2 controls the embryo-toseedling phase transition. PLoS Genet. 7, e1002014 (2011).

27. Jackson, J. P., Lindroth, A. M., Cao, X. \& Jacobsen, S. E. Control of CpNpG DNA methylation by the KRYPTONITE histone H3 methyltransferase. Nature 416, 556-560 (2002).

28. Zheng, J. et al. A novel role for histone methyltransferase KYP/SUVH4 in the control of Arabidopsis primary seed dormancy. New Phytol. 193, 605-616 (2012).

29. Cho, J. N. et al. Control of seed germination by light-induced histone arginine demethylation activity. Dev. Cell 22, 736-748 (2012)

30. Molitor, A. M., Bu, Z., Yu, Y. \& Shen, W. H. Arabidopsis AL PHD-PRC1 complexes promote seed germination through H3K4me3-to-H3K27me3 chromatin state switch in repression of seed developmental genes. PLoS Genet. 10, e1004091 (2014).

31. Van Zanten, M. et al. HISTONE DEACETYLASE 9 represses seedling traits in Arabidopsis thaliana dry seeds. Plant J. 80, 475-488 (2014).

32. Wang, Z. et al. Arabidopsis paired amphipathic helix proteins SNL1 and SNL2 redundantly regulate primary seed dormancy via abscisic acidethylene antagonism mediated by histone deacetylation. Plant Cell 25, 149-166 (2013).

33. Soeno, K. et al. Auxin biosynthesis inhibitors, identified by a genomics-based approach, provide insights into auxin biosynthesis. Plant Cell Physiol. 51, 524-536 (2010)

34. Campanoni, P. \& Nick, P. Auxin-dependent cell division and cell elongation. 1-Naphthaleneacetic acid and 2,4-dichlorophenoxyacetic acid activate different pathways. Plant Physiol. 137, 939-948 (2005).

35. Chapman, E. J. \& Estelle, M. Mechanism of auxin-regulated gene expression in plants. Annu. Rev. Genet. 43, 265-285 (2009).

36. Sliwinska, E., Bassel, G. W. \& Bewley, J. D. Germination of Arabidopsis thaliana seeds is not completed as a result of elongation of the radicle but of the adjacent transition zone and lower hypocotyl. J. Exp. Bot. 60, 3587-3594 (2009).

37. Yu, J. \& Wen, C. K. Arabidopsis aux1rcr1 mutation alters AUXIN RESISTANT1 targeting and prevents expression of the auxin reporter DR5:GUS in the root apex. J. Exp. Bot. 64, 921-933 (2013).

38. Swarup, R. et al. Localization of the auxin permease AUX1 suggests two functionally distinct hormone transport pathways operate in the Arabidopsis root apex. Genes Dev. 15, 2648-2653 (2001).

39. De Smet, I. et al. Auxin-dependent regulation of lateral root positioning in the basal meristem of Arabidopsis. Development 134, 681-690 (2007).

40. Charron, J. B., He, H., Elling, A. A. \& Deng, X. W. Dynamic landscapes of four histone modifications during deetiolation in Arabidopsis. Plant Cell 21, 3732-3748 (2009).

41. Peret, B. et al. AUX/LAX genes encode a family of auxin influx transporters that perform distinct functions during Arabidopsis development. Plant Cell 24, 2874-2885 (2012).

42. Masubelele, N. H. et al. D-type cyclins activate division in the root apex to promote seed germination in Arabidopsis. Proc. Natl Acad. Sci. USA 102, 15694-15699 (2005)

43. Dewitte, W. \& Murray, J. A. The plant cell cycle. Annu. Rev. Plant Biol. 54, 235-264 (2003).
44. Barroco, R. M. et al. The role of the cell cycle machinery in resumption of postembryonic development. Plant Physiol. 137, 127-140 (2005).

45. Toorop, P. E., Barroco, R. M., Engler, G., Groot, S. P. \& Hilhorst, H. W. Differentially expressed genes associated with dormancy or germination of Arabidopsis thaliana seeds. Planta 221, 637-647 (2005).

46. Debeaujon, I. \& Koornneef, M. Gibberellin requirement for Arabidopsis seed germination is determined both by testa characteristics and embryonic abscisic acid. Plant Physiol. 122, 415-424 (2000).

47. Kucera, B., Cohn, M. A. \& Leubner-Metzger, G. Plant hormone interactions during seed dormancy release and germination. Seed Sci. Res. 15, 281-307 (2005).

48. Yamaguchi, S. \& Kamiya, Y. Gibberellins and light-stimulated seed germination. J. Plant. Growth Regul. 20, 369-376 (2001).

49. Okamoto, M. et al. CYP707A1 and CYP707A2, which encode abscisic acid $8^{\prime}$-hydroxylases, are indispensable for proper control of seed dormancy and germination in Arabidopsis. Plant Physiol. 141, 97-107 (2006).

50. Bowen, A. J. et al. PAH-domain-specific interactions of the Arabidopsis transcription coregulator SIN3-LIKE1 (SNL1) with telomere-binding protein 1 and ALWAYS EARLY2 Myb-DNA binding factors. J. Mol. Biol. 395, 937-949 (2010).

51. Belin, C., Megies, C., Hauserova, E. \& Lopez-Molina, L. Abscisic acid represses growth of the Arabidopsis embryonic axis after germination by enhancing auxin signaling. Plant Cell 21, 2253-2268 (2009).

52. Band, L. R. et al. Systems analysis of auxin transport in the Arabidopsis root apex. Plant Cell 26, 862-875 (2014).

53. Vieten, A. et al. Functional redundancy of PIN proteins is accompanied by auxin-dependent cross-regulation of PIN expression. Development 132, 4521-4531 (2005).

54. Marchant, A. \& Bennett, M. J. The Arabidopsis AUX1 gene: a model system to study mRNA processing in plants. Plant Mol. Biol. 36, 463-471 (1998).

55. Blilou, I. et al. The PIN auxin efflux facilitator network controls growth and patterning in Arabidopsis roots. Nature 433, 39-44 (2005).

56. Swarup, R. et al. Structure-function analysis of the presumptive Arabidopsis auxin permease AUX1. Plant Cell 16, 3069-3083 (2004).

57. Ulmasov, T., Murfett, J., Hagen, G. \& Guilfoyle, T. J. Aux/IAA proteins repress expression of reporter genes containing natural and highly active synthetic auxin response elements. Plant Cell 9, 1963-1971 (1997).

58. Alonso-Blanco, C., Bentsink, L., Hanhart, C. J., Blankestijn-de Vries, H. \& Koornneef, M. Analysis of natural allelic variation at seed dormancy loci of Arabidopsis thaliana. Genetics 164, 711-729 (2003).

59. Nakabayashi, K., Okamoto, M., Koshiba, T., Kamiya, Y. \& Nambara, E. Genome-wide profiling of stored mRNA in Arabidopsis thaliana seed germination: epigenetic and genetic regulation of transcription in seed. Plant $J$. 41, 697-709 (2005).

60. Lin, Y., Pajak, A., Marsolais, F., McCourt, P. \& Riggs, C. D. Characterization of a cruciferin deficient mutant of Arabidopsis and its utility for overexpression of foreign proteins in plants. PLoS ONE 8, e64980 (2013).

61. Clough, S. J. \& Bent, A. F. Floral dip: a simplified method for Agrobacteriummediated transformation of Arabidopsis thaliana. Plant J. 16, 735-743 (1998).

62. Jefferson, R. A., Kavanagh, T. A. \& Bevan, M. W. GUS fusions: betaglucuronidase as a sensitive and versatile gene fusion marker in higher plants. EMBO J. 6, 3901-3907 (1987).

63. Mironova, V. V. et al. A plausible mechanism for auxin patterning along the developing root. BMC Syst. Biol. 4, 98 (2010).

64. Xiang, Y. et al. REDUCED DORMANCY5 Encodes a protein phosphatase 2C that is required for seed dormancy in Arabidopsis. Plant Cell 26, 4362-4375 (2014).

65. Chua, Y. L., Channeliere, S., Mott, E. \& Gray, J. C. The bromodomain protein GTE6 controls leaf development in Arabidopsis by histone acetylation at ASYMMETRIC LEAVES1. Genes Dev. 19, 2245-2254 (2005).

66. Edlund, A., Eklof, S., Sundberg, B., Moritz, T. \& Sandberg, G. A microscale technique for gas chromatography-mass spectrometry measurements of picogram amounts of indole-3-acetic acid in plant tissues. Plant Physiol. 108, 1043-1047 (1995).

67. Bowler, C. et al. Chromatin techniques for plant cells. Plant J. 39, 776-789 (2004).

68. Menges, M. \& Murray, J. A. Synchronous Arabidopsis suspension cultures for analysis of cell-cycle gene activity. Plant J. 30, 203-212 (2002).

\section{Acknowledgements}

We thank Dr Chikuang Wen for aux1-T seeds, Dr Jeff A. Long for the hda19 mutant seeds and Dr Youfa Cheng for the $y u c 3$ and $y u c 8$ seeds. We are also grateful to Dr Chin-Ta Lin for proofreading, and Suhua Yang and Zhen Xue for technical support. This project was supported by the National Basic Research Program of China (973 Program; grant No.2014CB943400) and the National Natural Science Foundation of China (No. 31171164 and No. 31371242) 


\section{Author contributions}

Y.L. and Z.W. designed the research; Z.W., F.C., H.C. and X.L. performed the experiments; Z.W. and Y.L. analysed the data; Z.W., M.D., C.Z., J.Z., C.X., J.X. and Y.L. contributed reagents/materials/analysis tools; Z.W., Y.L., X.D., Y.X. and W.J.J.S. contributed to the discussion and manuscript; Z.W. and Y.L. wrote the paper.

\section{Additional information}

Supplementary Information accompanies this paper at http://www.nature.com/ naturecommunications

Competing financial interests: The authors declare no competing financial interests.

Reprints and permission information is available online at http://npg.nature.com/ reprintsandpermissions/
How to cite this article: Wang, Z. et al. Arabidopsis seed germination speed is controlled by SNL histone deacetylase-binding factor-mediated regulation of AUX1.

Nat. Commun. 7, 13412 doi: 10.1038/ncomms13412 (2016).

Publisher's note: Springer Nature remains neutral with regard to jurisdictional claims in published maps and institutional affiliations.

(c) (i) This work is licensed under a Creative Commons Attribution 4.0 International License. The images or other third party material in this article are included in the article's Creative Commons license, unless indicated otherwise in the credit line; if the material is not included under the Creative Commons license, users will need to obtain permission from the license holder to reproduce the material To view a copy of this license, visit http://creativecommons.org/licenses/by/4.0/

(C) The Author(s) 2016 